\title{
Derived categories and the genus of space curves
}

\author{
Emanuele Macrì and Benjamin Schmidt
}

\begin{abstract}
We generalize a classical result about the genus of curves in projective space by Gruson and Peskine to principally polarized abelian threefolds of Picard rank one. The proof is based on wall-crossing techniques for ideal sheaves of curves in the derived category. In the process, we obtain bounds for Chern characters of other stable objects such as rank two sheaves. The argument gives a proof for projective space as well. In this case these techniques also indicate an approach for a conjecture by Hartshorne and Hirschowitz and we prove first steps toward it.
\end{abstract}

\section{Introduction}

A celebrated result in the theory of space curves is the following [Hal82, GP78, Har80].

Theorem 1.1 (Gruson-Peskine, Harris). Let $d, k>0$ and $g \geqslant 0$ be integers. Let $C \subset \mathbb{P}^{3}$ be an integral curve of degree $d$ and arithmetic genus $g$. Assume

- $H^{0}\left(\mathbb{P}^{3}, I_{C}(k-1)\right)=0$ and

- $d>k(k-1)$.

Then

$$
g \leqslant \frac{d^{2}}{2 k}+\frac{1}{2} d(k-4)+1-\varepsilon \quad \text { for } \quad \varepsilon=\frac{1}{2} f\left(k-f-1+\frac{f}{k}\right),
$$

where $d \equiv-f(\bmod k)$ and $0 \leqslant f<k$.

For example, if $k=1$, this says that the largest genus for a fixed degree is given by that of a plane curve; that is, $g \leqslant(d-1)(d-2) / 2$. For $k=2$, it corresponds to Castelnuovo's inequality for non-planar curves [Har77, Chapter IV, Theorem 6.4]. The first goal of this article is to prove a version of this theorem for other threefolds by using the theory of stability in the derived category. The second goal is to attack a conjecture by Hartshorne and Hirschowitz for $d \leqslant k(k-1)$ in the case of $\mathbb{P}^{3}$ with similar techniques.

Tilt stability. Given a curve $C \subset \mathbb{P}^{3}$, there are two exact sequences in the category of coherent sheaves associated with its ideal sheaf $I_{C}$. For a non-zero section of $H^{0}\left(\mathbb{P}^{3}, I_{C}(h)\right)$, we

Received 27 February 2018, accepted in final form 2 April 2019.

2010 Mathematics Subject Classification 14H50 (primary), 14F05, 14J30, 18E30 (secondary).

Keywords: space curves, stability conditions, derived categories, classical algebraic geometry.

This journal is (C) Foundation Compositio Mathematica 2020. This article is distributed with Open Access under the terms of the Creative Commons Attribution Non-Commercial License, which permits non-commercial reuse, distribution, and reproduction in any medium, provided that the original work is properly cited. For commercial re-use, please contact the Foundation Compositio Mathematica.

The authors were partially supported by the NSF grant DMS-1523496 and by the NSF FRG-grant DMS1664215. 


\section{E. MACRÌ AND B. SCHMIDT}

can simply consider the associated sequence

$$
0 \rightarrow \mathcal{O}_{\mathbb{P}^{3}}(-h) \rightarrow I_{C} \rightarrow F \rightarrow 0 .
$$

Otherwise, for a non-zero section of $H^{2}\left(\mathbb{P}^{3}, I_{C}(m-4)\right)=\operatorname{Ext}^{1}\left(\mathcal{I}_{C}, \mathcal{O}_{\mathbb{P}^{3}}(-m)\right)$, we can consider the corresponding extension

$$
0 \rightarrow \mathcal{O}_{\mathbb{P}^{3}}(-m) \rightarrow E \rightarrow I_{C} \rightarrow 0 .
$$

The genus of $C$ can be bounded, by bounding the Chern characters of both $E$ and $F$. Just using the first exact sequence is not enough to conclude the proof of Theorem 1.1, since the bound therein is not decreasing for $h$ large. The key observation in our approach is that $E$ can also be thought of as a subobject of the ideal sheaf $I_{C}$, but in a different abelian category. By using a notion of stability on these categories and by just taking the first factor of the HarderNarasimhan filtration of $I_{C}$ with respect to this stability, we can select a canonical sequence among all these.

More generally, let $X$ be a smooth projective threefold. We are going to use the notion of tilt stability. It is reviewed in Section 2. This is a weak stability condition in the bounded derived category of coherent sheaves on $X$, which was introduced in [BMT14] (based on Bridgeland stability on surfaces [Bri08, AB13]). It can be thought of as a generalization of the classical notion of slope stability for sheaves on surfaces. If we fix an ample divisor $H$ on $X$, it roughly amounts to replacing the category of coherent sheaves with the heart of a bounded t-structure $\operatorname{Coh}^{\beta}(X)$ in the bounded derived category $\mathrm{D}^{\mathrm{b}}(X)$ and the classical slope with a new slope function $\nu_{\alpha, \beta}$. Everything depends on two real parameters $\alpha, \beta \in \mathbb{R}$ with $\alpha>0$. The starting point for us is that for $\alpha \gg 0$ and $\beta<0$, and for any curve $C \subset X$, the ideal sheaf $\mathcal{I}_{C}$ is $\nu_{\alpha, \beta}$-stable (see Lemma 2.5 for details). The key idea is to study variation of stability for $\mathcal{I}_{C}$ with to respect to $\alpha$ and $\beta$.

The main theorem. Let $X$ be a smooth projective threefold of Picard rank one; that is, its Néron-Severi group is generated by the class of a single ample divisor $H$. For a subvariety $Y \subset X$ of dimension $n=1,2$, we define its degree as $H^{n} \cdot Y / H^{3}$. We also define an extension of the remainder term in Theorem 1.1 as follows. For a rational number $d \in \frac{1}{2} \mathbb{Z}$ and an integer $k \geqslant 1$, we set

$$
\varepsilon(d, k)=\frac{1}{2} f\left(k-f-1+\frac{f}{k}\right)+\varepsilon(d, 1) \quad \text { for } \quad \varepsilon(d, 1)= \begin{cases}1 / 24 & \text { if } d \notin \mathbb{Z}, \\ 0 & \text { if } d \in \mathbb{Z},\end{cases}
$$

where $d \equiv-f(\bmod k)$ and $0 \leqslant f<k$ with $f \in \frac{1}{2} \mathbb{Z}$.

Theorem 1.2 (See Theorem 3.1). Assume that $X$ satisfies Assumptions A, B, C given below. Let $k \in \mathbb{Z}_{>0}$ and $d \in \frac{1}{2} \mathbb{Z}_{>0}$, and let $C \subset X$ be an integral curve of degree $d$. Further, assume

- $H^{0}\left(X, I_{C}\left((k-1) H^{\prime}\right)=0\right.$ for any divisor $H^{\prime}$ in the same numerical class as $H$, and

- $d>k(k-1)$.

Then

$$
\frac{\operatorname{ch}_{3}\left(\mathcal{I}_{C}\right)}{H^{3}} \leqslant E(d, k):=\frac{d^{2}}{2 k}+\frac{d k}{2}-\varepsilon(d, k) .
$$

For example, in characteristic zero, the assumptions of Theorem 1.2 are satisfied in the case of $\mathbb{P}^{3}$, principally polarized abelian threefolds of Picard rank one, and index two Fano threefolds of Picard rank one with degree one or two. In fact, the case of $\mathbb{P}^{3}$ is independent of the characteristic of the field, and Theorem 1.1 holds in that case. We note that already the case $k=1$ strengthens 


\section{DERIVED CATEGORIES AND THE GENUS OF SPACE CURVES}

a conjecture by Debarre [Deb94, Section 5] and a result by Pareschi-Popa [PP08, Theorem B] for the special case of principally polarized abelian threefolds of Picard rank one (see [LN16] for results on general polarized abelian varieties). The precise assumptions are the following.

Assumption A. The Néron-Severi group is generated by the class of an ample divisor $H$. Moreover, the Chern character of any $E \in \operatorname{Coh}(X)$ satisfies $\operatorname{ch}_{2}(E) \in \frac{1}{2} H^{2} \cdot \mathbb{Z}$ and $\operatorname{ch}_{3}(E) \in$ $\frac{1}{6} H^{3} \cdot \mathbb{Z}$.

Without the part about $\operatorname{ch}_{2}(E)$ in Assumption A, curves of small degree pose issues. Some bounds can be proved without this assumption, but they do not seem optimal. However, the Picard rank one assumption is more important in our argument, since without it determining the tilt stability of ideal sheaves becomes substantially more involved. The part about $\operatorname{ch}_{3}(E)$ is for computational reasons to bound the Chern characters of rank two sheaves.

Assumption B. Any slope semistable sheaf $E \in \operatorname{Coh}(X)$ satisfies

$$
\Delta(E):=\frac{\left(H^{2} \cdot \operatorname{ch}_{1}(E)\right)^{2}-2\left(H^{3} \cdot \operatorname{ch}_{0}(E)\right)\left(H \cdot \operatorname{ch}_{2}(E)\right)}{\left(H^{3}\right)^{2}} \geqslant 0 .
$$

Assumption B is well known to be true in characteristic zero, being a consequence of the classical Bogomolov inequality [Rei78, Bog79, Gie79]. In positive characteristic, it is only sometimes satisfied, for example for $\mathbb{P}^{3}$ and abelian threefolds (see [Lan04] for more details).

For any $\beta \in \mathbb{R}$ and $E \in \mathrm{D}^{\mathrm{b}}(X)$, we define the twisted Chern character $\operatorname{ch}^{\beta}(E):=\operatorname{ch}(E) \cdot e^{-\beta H}$. Note that for $\beta \in \mathbb{Z}$, this is simply saying $\operatorname{ch}^{\beta}(E)=\operatorname{ch}(E(-\beta H))$.

Assumption C. For any $\nu_{\alpha, \beta}$-semistable object $E \in \operatorname{Coh}^{\beta}(X)$, the inequality

$$
Q_{\alpha, \beta}(E):=\alpha^{2} \Delta(E)+\frac{4\left(H \cdot \operatorname{ch}_{2}^{\beta}(E)\right)^{2}}{\left(H^{3}\right)^{2}}-\frac{6\left(H^{2} \cdot \operatorname{ch}_{1}^{\beta}(E)\right) \operatorname{ch}_{3}^{\beta}(E)}{\left(H^{3}\right)^{2}} \geqslant 0
$$

holds.

Assumption $\mathrm{C}$ is the crucial ingredient in the proof. It roughly tells us that the ideal sheaf of a curve of large genus has to be destabilized at a certain point, and it allows us to reduce the number of possible walls. This assumption is part of a more general conjecture in [BMT14, BMS16] for characteristic zero. The case of $\mathbb{P}^{3}$ was shown in [Mac14b], and the proof actually works in any characteristic. The smooth quadric hypersurface in $\mathbb{P}^{4}$ was done in [Sch14]. Later, both of these were generalized to Fano threefolds of Picard rank one in [Li19]. Moreover, the case of abelian threefolds were handled independently in [MP15, MP16] and [BMS16], and CalabiYau threefolds of abelian type in [BMS16]. Most recently, it was shown in [Kos18] for the case of $\mathbb{P}^{2} \times E, \mathbb{P}^{1} \times \mathbb{P}^{1} \times E$, and $\mathbb{P}^{1} \times A$, where $E$ is an arbitrary elliptic curve and $A$ is an arbitrary abelian surface. For higher Picard rank, it is known to be false in general. Counterexamples were given in [Sch17, Kos18, MS19]. In general, a relation between Assumption C and Castelnuovo theory for projective curves [Har82a, CCD93] was already observed in [BMT14, Tra14].

Strategy of the proof. The general idea of the proof of Theorem 1.2 is to study potential walls in tilt stability in the $(\alpha, \beta)$-plane for the ideal sheaf of a curve $C \subset X$, namely codimension one loci at which stability changes. By the Hirzebruch-Riemann-Roch theorem, bounding $\operatorname{ch}_{3}\left(\mathcal{I}_{C}\right)$ is equivalent to bounding the genus. As mentioned previously, Assumption $\mathrm{C}$ implies that there has to be at least one wall. For each wall, there exists a semistable subobject $E$ with semistable 


\section{E. MACRÌ AND B. SCHMIDT}

quotient $G$. Bounding the third Chern character for $E$ and $G$ induces a bound for $\operatorname{ch}_{3}\left(\mathcal{I}_{C}\right)$. In order to bound the Chern characters of $E$ and $G$, we study tilt stability for these objects. It turns out that $\Delta(E), \Delta(G)<\Delta\left(\mathcal{I}_{C}\right)$, and since these numbers are non-negative integers, this process has to terminate.

To unify the notation among different $X$, we set

$$
H \cdot \operatorname{ch}(E):=\left(\frac{H^{3} \cdot \operatorname{ch}_{0}(E)}{H^{3}}, \frac{H^{2} \cdot \operatorname{ch}_{1}(E)}{H^{3}}, \frac{H \cdot \operatorname{ch}_{2}(E)}{H^{3}}, \frac{\operatorname{ch}_{3}(E)}{H^{3}}\right) .
$$

The condition $d>k(k-1)$ implies that this process only requires the study of three types of objects which are handled in the next three statements.

Proposition 1.3 (See Proposition 3.2). Let $E \in \mathrm{Coh}^{\beta}(X)$ be a $\nu_{\alpha, \beta}$-semistable object for some $(\alpha, \beta)$ with either $H \cdot \operatorname{ch}(E)=(1,0,-d, e)$ or $H \cdot \operatorname{ch}(E)=(-1,0, d, e)$. Then

$$
e \leqslant \frac{d(d+1)}{2}-\varepsilon(d, 1)=E(d, 1) \text {. }
$$

If $E$ is an ideal sheaf of a curve, then Proposition 1.3 is the $k=1$ version of Theorem 1.2. Using derived duals (see Proposition 2.6 for details), it is only necessary to prove the case of positive rank.

Theorem 1.4 (See Theorem 3.4). Let $E \in \operatorname{Coh}^{\beta}(X)$ be a $\nu_{\alpha, \beta}$-semistable object for some $(\alpha, \beta)$ with $H \cdot \operatorname{ch}(E)=(0, c, d, e)$, where $c>0$. Then

$$
e \leqslant \frac{c^{3}}{24}+\frac{d^{2}}{2 c}-\varepsilon\left(d+\frac{c^{2}}{2}, c\right) .
$$

The case $c=1$ for Theorem 1.4 was proved for $\mathbb{P}^{3}$ in [Sch15, Lemma 5.4].

Theorem 1.5 (See Theorem 3.6). Let $E \in \operatorname{Coh}^{\beta}(X)$ be a $\nu_{\alpha, \beta}$-semistable object for some $(\alpha, \beta)$ with $H \cdot \operatorname{ch}(E)=(2, c, d, e)$.

(i) If $c=-1$, then $d \leqslant 0$ and $e \leqslant \frac{1}{2} d^{2}-d+\frac{5}{24}-\varepsilon\left(d-\frac{1}{2}, 1\right)$.

(ii) If $c=0$, then $d \leqslant 0$.

(a) If $d=0$, then $e \leqslant 0$.

(b) If $d=-\frac{1}{2}$, then $e \leqslant \frac{1}{6}$.

(c) If $d \leqslant-1$, then $e \leqslant \frac{1}{2} d^{2}+\frac{5}{24}-\varepsilon\left(d+\frac{1}{2}, 1\right)$.

If $X=\mathbb{P}^{3}$ and $c=-1$, Theorem 1.5 implies the corresponding case of Theorem 1.10 by Hartshorne and Hirschowitz even without the reflexiveness hypothesis. The case $c=0$ gives a weaker bound here. For just $\mathbb{P}^{3}$, we could get the stronger bound by a more careful analysis, but it turns out to be wrong for more general threefolds.

All of these statements, including Theorem 1.2, are proved with the following strategy. Let $E$ be the object for which we want to bound $\operatorname{ch}_{3}(E)$. We start by proving the statement for small values of $\Delta(E)$ using $Q_{\alpha, \beta}(E) \geqslant 0$ whenever $E$ is $\nu_{\alpha, \beta}$-semistable. For larger values of $\Delta(E)$, this strategy provides non-optimal bounds [BMT14, Mac14b, Sun18, Sun19]. Instead, we study wall-crossing via the following steps. Assume that $\operatorname{ch}_{3}(E)$ is larger than expected. As explained before, we can assume $\operatorname{ch}_{0}(E) \geqslant 0$.

(1) Besides implying the existence of a destabilizing wall, the inequality $Q_{\alpha, \beta}(E) \geqslant 0$ gives a bound on the rank of the destabilizing subobject (see Lemma 2.4 for details). For example, 


\section{DERIVED CATEGORIES AND THE GENUS OF SPACE CURVES}

for ideal sheaves of curves satisfying the assumptions of Theorem 1.2, the subobject can only be of rank one or two. Let

$$
0 \rightarrow F \rightarrow E \rightarrow G \rightarrow 0
$$

be the destabilizing sequence. The argument is always symmetric in $F$ and $G$, and without loss of generality, we can assume $\operatorname{ch}_{0}(F) \geqslant 1$.

(2) Using the fact that $\operatorname{ch}_{1}^{\beta}(F)>0$ and $\operatorname{ch}_{1}^{\beta}(G)>0$ for any $\beta$ along the wall, we obtain a lower and upper bound on $\operatorname{ch}_{1}(F)$.

(3) The Bogomolov inequality $\Delta(F) \geqslant 0$ yields an upper bound on $\operatorname{ch}_{2}(F)$. The inequality $\Delta(G) \geqslant 0$ yields another bound on $\operatorname{ch}_{2}(F)$ (lower or upper bound depending on the rank of $G)$. Moreover, the fact that a wall cannot lie in the area $Q_{\alpha, \beta}(E)<0$ leads to a lower bound on $\mathrm{ch}_{2}(F)$. Overall, this reduces the problem to finitely many walls.

(4) Next, we use some previously obtained bounds for $\operatorname{ch}_{3}(F)$ and $\operatorname{ch}_{3}(G)$ to bound $\operatorname{ch}_{3}(E)$.

(5) In general, the walls are linearly ordered. The last step is to check that the previous bound is decreasing with this ordering, and the largest wall still provides a contradiction.

We prove the statements in the following order. First, the case $c=1$ in Theorem 1.4 is proved via $Q_{\alpha, \beta}(E) \geqslant 0$ (see Lemma 3.3 for details). Next, we prove Proposition 1.3. It turns out that the subobjects are also of rank one, for which we use induction, and the bounds on the quotients follow from the $c=1$ case in Theorem 1.4. After that, we use Proposition 1.3 on both subobjects and quotients to prove Theorem 1.4. All of the previous statements are used to prove Theorem 1.5 with an induction on $\Delta(E)$. Finally, Theorem 1.2 can be proved using the same steps again.

The Hartshorne-Hirschowitz conjecture. Coming back to the case of projective space, our aim is to improve our techniques toward a possible approach to the Hartshorne-Hirschowitz conjecture, namely to the case $d \leqslant k(k-1)$. Let us first recall the statement of the conjecture [Har87, HH88, Har88].

For given integers $d$ and $k$, let $G(d, k)$ be the maximal genus of an integral curve $C \subset \mathbb{P}^{3}$ with degree $d$ such that $C$ is not contained in a surface of degree less than $k$. It is easy to check that $d \geqslant \frac{1}{6}\left(k^{2}+4 k+6\right)$. For $d>k(k-1)$, the genus $G(d, k)$ is given by Theorem 1.1 , since a curve with genus $G(d, k)$ always exists under those assumptions. If

$$
\frac{1}{6}\left(k^{2}+4 k+6\right) \leqslant d<\frac{1}{3}\left(k^{2}+4 k+6\right)
$$

then it is not hard to find a bound from above for $G(d, k)$, but currently it is still not known in full generality if this bound is sharp (see $\left[\mathrm{Har} 87, \mathrm{BBE}^{+} 97\right]$ for results in this direction). We are interested in the remaining case

$$
\frac{1}{3}\left(k^{2}+4 k+6\right) \leqslant d \leqslant k(k-1) .
$$

Note that this case only makes sense for $k \geqslant 5$. We first introduce another error term as follows. For any integer $c \in \mathbb{Z}$, let

$$
\delta(c):= \begin{cases}3 & \text { if } c=1,3, \\ 1 & \text { if } c \equiv 2(\bmod 3), \\ 0 & \text { otherwise }\end{cases}
$$




\section{E. MACRÌ AND B. SCHMIDT}

Then, for any integers $k \geqslant 5$ and $f \in[k-1,2 k-5]$, we define integers

$$
\begin{aligned}
& A(k, f):=\frac{1}{3}\left(k^{2}-k f+f^{2}-2 k+7 f+12+\delta(2 k-f-6)\right), \\
& B(k, f):=\frac{1}{3}\left(k^{2}-k f+f^{2}+6 f+11+\delta(2 k-f-7)\right) .
\end{aligned}
$$

A straightforward computation shows that $A(k, f)$ is an increasing function for $f \in[k-1$, $2 k-5]$ and that it partitions our range of $d$ in $(1.1): A(k, k-1)=\left\lceil\frac{1}{3}\left(k^{2}+4 k+6\right)\right\rceil$ and $A(k, 2 k-5)=k(k-1)+1$. Moreover, we have $A(k, f)<B(k, f) \leqslant A(k, f+1)$.

Conjecture 1.6 (Hartshorne-Hirschowitz, see Conjecture 4.1). Let $d, k>0$ be integers. Assume $A(k, f) \leqslant d<A(k, f+1)$ for some $f \in[k-1,2 k-6]$. Then

$$
G(d, k)=d(k-1)+1-\left(\begin{array}{c}
k+2 \\
3
\end{array}\right)+\left(\begin{array}{c}
f-k+4 \\
3
\end{array}\right)+h(d),
$$

where

$$
h(d)= \begin{cases}0 & \text { if } A(k, f) \leqslant d \leqslant B(k, f), \\ \frac{1}{2}(d-B(k, f))(d-B(k, f)+1) & \text { if } B(k, f) \leqslant d<A(k, f+1)) .\end{cases}
$$

By [HH88], it is known that there exist curves with genus $G(d, k)$ satisfying the hypothesis of the conjecture. Therefore, one only has to prove that every curve satisfies this bound. This is known for a few values of $f$ : the cases $f=k-1, k$ were proved in [Har88], while the case $f=2 k-6$ is in [GP83], $f=2 k-7$ in [El191], $f=2 k-8,2 k-9$ in [ES92], and $f=2 k-10$ in [Str90].

This conjecture is partially based on the fact that this bound is obtained for curves with an extension

$$
0 \rightarrow \mathcal{O}_{\mathbb{P}^{3}}(-f-4) \rightarrow E \rightarrow \mathcal{I}_{C} \rightarrow 0
$$

by bounding the third Chern character of the reflexive sheaf $E$. This sequence constitutes a potential wall in tilt stability for $\mathcal{I}_{C}$ because in our abelian category, this corresponds to an exact sequence

$$
0 \rightarrow E \rightarrow \mathcal{I}_{C} \rightarrow \mathcal{O}_{\mathbb{P}^{3}}(-f-4)[1] \rightarrow 0
$$

It turns out that our approach requires to study walls above or below this wall with slightly different methods, and therefore, we suggest the following two questions. We need one extra bit of notation (see Theorem 2.2 for a more detailed description of walls and their possible shapes). Given two elements $E, F \in \mathrm{D}^{\mathrm{b}}\left(\mathbb{P}^{3}\right)$, let $W(E, F)$ be the locus in the $(\alpha, \beta)$-plane where $E$ and $F$ have the same $\nu_{\alpha, \beta}$-slope. In the cases we will be interested in, these loci are semicircles with center on the $\beta$-axis.

Question 1.7. Assume the hypothesis of Conjecture 1.6. Let $C$ be an integral curve of genus $g$ and degree $d$ such that $H^{0}\left(\mathcal{I}_{C}(k-1)\right)=0$. If $\mathcal{I}_{C}$ is destabilized in tilt stability above or at the numerical wall $W\left(\mathcal{I}_{C}, \mathcal{O}(-f-4)[1]\right)$, does $g \leqslant G(d, k)$ hold?

Our second main result is an affirmative answer to this question in a smaller range.

Theorem 1.8 (See Theorem 4.2). Question 1.7 has an affirmative answer if $A(k, f) \leqslant d \leqslant B(k, f)$ and the base field has characteristic zero.

A full proof of the conjecture also requires studying walls below $W\left(\mathcal{I}_{C}, \mathcal{O}(-f-4)[1]\right)$. We suggest the following approach.

Question 1.9. Assume the hypothesis of Conjecture 1.6, and let $C$ be destabilized below the wall $W\left(\mathcal{I}_{C}, \mathcal{O}(-f-4)[1]\right)$. Is the maximal possible genus of $C$ decreasing with the size of the wall? 


\section{DERIVED CATEGORIES AND THE GENUS OF SPACE CURVES}

All arguments in Section 3 suggest that the maximum $\mathrm{ch}_{3}$ for semistable objects is decreasing with the size of the wall even beyond ideal sheaves. The most serious obstacle for studying this question is the fact that, in general, destabilizing subobjects can be reflexive sheaves of high rank. Beyond rank two, results are scarce. Another problem is that we would need to consider more general bounds for not necessarily integral curves, but in our setting this is probably more approachable. In any case, a positive answer to both Question 1.7 and Question 1.9 would indeed prove Conjecture 1.6 since if $C$ does not satisfy the conjecture, then $I_{C}$ will be destabilized at a certain point.

In order to handle rank two objects in the proof of Theorem 1.8, we need the following result [Har82b, Theorem 0.1], [GP83], [Har87, Theorems 3.2 and 3.3], [HH88], and [Har88, Theorem 1.1].

Theorem 1.10. Assume that the base field has characteristic zero. Let $E \in \operatorname{Coh}\left(\mathbb{P}^{3}\right)$ be a rank two reflexive sheaf with $\operatorname{ch}(E)=(2, c, d, e), c \geqslant-1$, and $H^{0}(E)=0$. Then $d \leqslant \frac{1}{6} c^{2}-\frac{2}{3} c-1-\frac{1}{3} \delta(c)$. Moreover,

(i) if $\frac{1}{6} c^{2}-c-\frac{8}{3}-\frac{1}{3} \delta(c-1) \leqslant d \leqslant \frac{1}{6} c^{2}-\frac{2}{3} c-1-\frac{1}{3} \delta(c)$, then $h^{2}(E)=0$ and

$$
e \leqslant-\frac{11}{6} c-2 d-2 ;
$$

(ii) if $d \leqslant \frac{1}{6} c^{2}-c-\frac{8}{3}-\frac{1}{3} \delta(c-1)$, then

$$
h^{2}(E) \leqslant \frac{\left(c^{2}-6 c-6 d-2 \delta(c-1)-10\right)\left(c^{2}-6 c-6 d-2 \delta(c-1)-16\right)}{72}
$$

and

$$
e \leqslant \frac{c^{4}}{72}-\frac{c^{3}}{6}+\frac{5 c^{2}}{36}+\frac{c}{3}-\frac{c^{2} d}{6}+c d+\frac{d^{2}}{2}+\frac{d}{6}+\frac{2}{9}-\frac{\delta(c-1)}{18}\left(c^{2}-6 c-6 d-\delta(c-1)-13\right) .
$$

Furthermore, these bounds are strict in the sense that there are rank two stable reflexive sheaves $E$ with $H^{0}(E)=0$ reaching them in all cases.

A more detailed tilt stability version of Theorem 1.10 is surely necessary to answer Questions 1.7 and 1.9 in general.

Finally, we illustrate our approach in one example. In Proposition 4.10, we prove Conjecture 1.6 in the case $d=A(k, 2 k-11)$ when $k \geqslant 31$. For a fixed $k$, this is the largest degree for which the conjecture is unknown. We have no doubt that a slightly more careful analysis would also handle the cases $k<31$.

\section{Notation}

$$
\begin{aligned}
X & \text { smooth projective threefold over an algebraically closed field } \mathbb{F} \\
H & \text { fixed ample divisor on } X \\
\mathrm{D}^{\mathrm{b}}(X) & \text { bounded derived category of coherent sheaves on } X \\
\mathcal{H}^{i}(E) & \text { ith cohomology group of a complex } E \in \mathrm{D}^{\mathrm{b}}(X) \\
H^{i}(E) & \text { ith sheaf cohomology group of a complex } E \in \mathrm{D}^{\mathrm{b}}(X) \\
\mathbb{D}(\cdot) & \text { derived dual } \mathbf{R} \mathcal{H} \text { om }\left(\cdot, \mathcal{O}_{X}\right)[1] \\
\operatorname{ch}(E) & \text { Chern character of an object } E \in \mathrm{D}^{\mathrm{b}}(X) \\
\operatorname{ch}_{\leqslant l}(E) & \left(\operatorname{ch}_{0}(E), \ldots, \operatorname{ch}_{l}(E)\right) \\
H \cdot \operatorname{ch}(E) & \left(\frac{H^{3} \cdot \operatorname{ch}_{0}(E)}{H^{3}}, \frac{H^{2} \cdot \operatorname{ch}_{1}(E)}{H^{3}}, \frac{H \cdot \operatorname{ch}_{2}(E)}{H^{3}}, \frac{\operatorname{ch}_{3}(E)}{H^{3}}\right) \\
H \cdot \operatorname{ch}_{\leqslant l}(E) & \left(\frac{H^{3} \cdot \operatorname{ch}_{0}(E)}{H^{3}}, \ldots, \frac{H^{3-l} \cdot \operatorname{ch}_{l}(E)}{H^{3}}\right)
\end{aligned}
$$




\section{E. MACRÌ AND B. SCHMIDT}

\section{Background on stability conditions}

In [BMT14], the notion of tilt stability was introduced as an auxiliary notion in between slope stability and a conjectural construction of Bridgeland stability on threefolds. It turns out to be useful in its own right, as pointed out, for example, in [Sch15, Xia18]. In this section, we give a quick introduction to tilt stability and its basic properties. We will restrict to the case of Picard rank one, even though the theory can be developed more generally.

\subsection{Definition}

Let $X$ be a smooth projective threefold over an algebraically closed field $\mathbb{F}$. The first assumption we will make in this article is to restrict its possible divisors and curves.

Assumption A. The Néron-Severi group is generated by the class of an ample divisor $H$; that is,

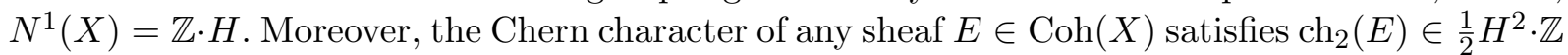
and $\operatorname{ch}_{3}(E) \in \frac{1}{6} H^{3} \cdot \mathbb{Z}$.

This assumption is not needed for the results in this preliminary section, but it will be important for the remainder of the article. It holds in particular for $\mathbb{P}^{3}$, for principally polarized abelian threefolds of Picard rank one, and for Fano threefolds of Picard rank one, index two, and degree one or two.

The classical slope for a coherent sheaf $E \in \operatorname{Coh}(X)$ is defined as

$$
\mu(E):=\frac{H^{2} \cdot \operatorname{ch}_{1}(E)}{H^{3} \cdot \operatorname{ch}_{0}(E)}
$$

where division by zero is interpreted as $+\infty$. A coherent sheaf $E$ is called slope (semi)stable if for any non-trivial proper subsheaf $F \subset E$, the inequality $\mu(F)<(\leqslant) \mu(E / F)$ holds. We will assume that the following assertion holds. In characteristic zero this is nothing but a consequence of the classical Bogomolov inequality [Rei78, Bog79, Gie79]. In positive characteristic, it holds, for example, in $\mathbb{P}^{3}$ and abelian threefolds [MR83, Lan04]. ${ }^{1}$

Assumption B. Any slope semistable sheaf $E \in \operatorname{Coh}(X)$ satisfies

$$
\Delta(E):=\frac{\left(H^{2} \cdot \operatorname{ch}_{1}(E)\right)^{2}-2\left(H^{3} \cdot \operatorname{ch}_{0}(E)\right)\left(H \cdot \operatorname{ch}_{2}(E)\right)}{\left(H^{3}\right)^{2}} \geqslant 0 .
$$

Note that, by using Assumption $\mathrm{A}$, we have $\Delta(E) \in \mathbb{Z}$. Let $\beta$ be an arbitrary real number. Then the twisted Chern character $\mathrm{ch}^{\beta}$ is defined to be $e^{-\beta H} \cdot \operatorname{ch}$. Explicitly,

$$
\begin{aligned}
& \operatorname{ch}_{0}^{\beta}=\operatorname{ch}_{0}, \operatorname{ch}_{1}^{\beta}=\operatorname{ch}_{1}-\beta H \cdot \operatorname{ch}_{0}, \quad \operatorname{ch}_{2}^{\beta}=\operatorname{ch}_{2}-\beta H \cdot \operatorname{ch}_{1}+\frac{1}{2} \beta^{2} H^{2} \cdot \operatorname{ch}_{0}, \\
& \operatorname{ch}_{3}^{\beta}=\operatorname{ch}_{3}-\beta H \cdot \operatorname{ch}_{2}+\frac{1}{2} \beta^{2} H^{2} \cdot \operatorname{ch}_{1}-\frac{1}{6} \beta^{3} H^{3} \cdot \operatorname{ch}_{0} .
\end{aligned}
$$

The process of tilting is used to construct a new heart of a bounded t-structure. For more information on the general theory of tilting, we refer to [HRS96, BvdB03]. A torsion pair is defined by

$$
\begin{aligned}
& \mathcal{T}_{\beta}:=\{E \in \operatorname{Coh}(X): \text { any quotient } E \rightarrow G \text { satisfies } \mu(G)>\beta\}, \\
& \mathcal{F}_{\beta}:=\{E \in \operatorname{Coh}(X): \text { any subsheaf } F \subset E \text { satisfies } \mu(F) \leqslant \beta\} .
\end{aligned}
$$

\footnotetext{
${ }^{1}$ In [Lan04], a general Bogomolov inequality is proved over any field, by adding an extra term to the inequality in Assumption B. However, this is generally not enough to define tilt stability.
} 


\section{DERIVED CATEGORIES AND THE GENUS OF SPACE CURVES}

The heart of a bounded t-structure is given as the extension closure $\operatorname{Coh}^{\beta}(X):=\left\langle\mathcal{F}_{\beta}[1], \mathcal{T}_{\beta}\right\rangle$. Let $\alpha>0$ be a positive real number. The tilt slope is defined as

$$
\nu_{\alpha, \beta}:=\frac{H \cdot \operatorname{ch}_{2}^{\beta}-\frac{1}{2} \alpha^{2} H^{3} \cdot \operatorname{ch}_{0}^{\beta}}{H^{2} \cdot \operatorname{ch}_{1}^{\beta}} .
$$

Similarly as before, an object $E \in \operatorname{Coh}^{\beta}(X)$ is called tilt-(semi)stable (or $\nu_{\alpha, \beta}$-(semi) stable) if for any non-trivial proper subobject $F \subset E$, the inequality $\nu_{\alpha, \beta}(F)<(\leqslant) \nu_{\alpha, \beta}(E / F)$ holds. Assumption $B$ implies that this notion of stability is well defined and that it shares many properties with slope stability for sheaves [BMT14]; in particular, Harder-Narasimhan filtrations exist and stability is open for varying $(\alpha, \beta)$.

\subsection{Walls and inequalities}

A version of the classical Bogomolov inequality also holds in tilt stability assuming that it holds for slope semistable sheaves.

Theorem 2.1 (Bogomolov inequality for tilt stability, [BMT14, Corollary 7.3.2]). Assume that Assumption $B$ holds. Then, any $\nu_{\alpha, \beta}$-semistable object $E \in \operatorname{Coh}^{\beta}(X)$ satisfies $\Delta(E) \geqslant 0$.

The following inequality involving the third Chern character was conjectured in [BMT14] and was brought into the following form in [BMS16].

Assumption C. For any $\nu_{\alpha, \beta}$-semistable object $E \in \operatorname{Coh}^{\beta}(X)$, the following inequality holds:

$$
Q_{\alpha, \beta}(E):=\alpha^{2} \Delta(E)+\frac{4\left(H \cdot \operatorname{ch}_{2}^{\beta}(E)\right)^{2}}{\left(H^{3}\right)^{2}}-\frac{6\left(H^{2} \cdot \operatorname{ch}_{1}^{\beta}(E)\right) \operatorname{ch}_{3}^{\beta}(E)}{\left(H^{3}\right)^{2}} \geqslant 0 .
$$

In our setting of Picard rank one, this is known to hold in characteristic zero ${ }^{2}$ for both Fano threefolds [Mac14b, Sch14, Li19] and abelian threefolds [MP15, MP16, BMS16].

Let $\Lambda \subset \mathbb{Z} \oplus \mathbb{Z} \oplus \frac{1}{2} \mathbb{Z}$ be the image of the map $H \cdot \operatorname{ch}_{\leqslant 2}$. Notice that $\nu_{\alpha, \beta}$ factors through $H \cdot \operatorname{ch} \leqslant 2$. Varying $(\alpha, \beta)$ changes the set of stable objects. A numerical wall in tilt stability with respect to a class $v \in \Lambda$ is a non-trivial proper subset $W$ of the upper half plane given by an equation of the form $\nu_{\alpha, \beta}(v)=\nu_{\alpha, \beta}(w)$ for another class $w \in \Lambda$. We will usually write $W=W(v, w)$.

A subset $S$ of a numerical wall $W$ is called an actual wall if the set of semistable objects with class $v$ changes at $S$. The structure of walls in tilt stability is rather simple. Parts (i)-(iv) of Theorem 2.2 are usually called Bertram's nested wall theorem and appeared in [Mac14a], while parts (v) and (vi) can be found in [BMS16, Appendix A].

Theorem 2.2 (Structure theorem for walls in tilt stability). Let $v \in \Lambda$ be a fixed class. All numerical walls in the following statements are with respect to $v$.

(i) Numerical walls in tilt stability are either semicircles with center on the $\beta$-axis or rays parallel to the $\alpha$-axis. Moreover, a semicircular wall with radius $\rho$ and center $s$ satisfies

$$
\frac{\left(H^{3} \cdot \operatorname{ch}_{0}(v)\right)^{2}}{\left(H^{3}\right)^{2}} \rho^{2}+\Delta(v)=\frac{\left(H^{3} \cdot \operatorname{ch}_{0}(v) s-H^{2} \cdot \operatorname{ch}_{1}(v)\right)^{2}}{\left(H^{3}\right)^{2}} .
$$

If $v_{0} \neq 0$, there is exactly one numerical vertical wall given by $\beta=v_{1} / v_{0}$. If $v_{0}=0$, there is no actual vertical wall.

${ }^{2}$ Some of the arguments in the known proofs do generalize directly to positive characteristic. For example, in $\mathbb{P}^{3}$ Assumption $\mathrm{C}$ holds over any field. 


\section{E. MACRÌ AND B. SCHMIDT}

(ii) The curve $\nu_{\alpha, \beta}(v)=0$ is given by a hyperbola, which may be degenerate. Moreover, this hyperbola intersects all semicircular walls at their top point.

(iii) If two numerical walls given by classes $w, u \in \Lambda$ intersect, then $v, w$ and $u$ are linearly dependent. In particular, the two walls are completely identical.

(iv) If a numerical wall has a single point at which it is an actual wall, then all of it is an actual wall.

(v) If there is an actual wall numerically defined by an exact sequence of tilt semistable objects $0 \rightarrow F \rightarrow E \rightarrow G \rightarrow 0$ such that $H \cdot \operatorname{ch}_{\leqslant 2}(E)=v$, then

$$
\Delta(F)+\Delta(G) \leqslant \Delta(E) .
$$

Moreover, equality holds if and only if $H \cdot \operatorname{ch}_{\leqslant 2}(G)=0$.

(vi) If $\Delta(E)=0$, then $E$ can only be destabilized at the unique numerical vertical wall. In particular, shifts of line bundles are tilt semistable everywhere.

If $W=W(v, w)$ is a semicircular wall in tilt stability for two numerical classes $v, w \in \Lambda$, then we denote its radius by $\rho_{W}=\rho(v, w)$ and its center on the $\beta$-axis by $s_{W}=s(v, w)$. The structure of the locus $Q_{\alpha, \beta}(E)=0$ fits right into this picture; indeed, a straightforward computation shows the following.

Lemma 2.3. Let $E \in \mathrm{D}^{\mathrm{b}}(X)$. The equation $Q_{\alpha, \beta}(E)=0$ is equivalent to

$$
\nu_{\alpha, \beta}(E)=\nu_{\alpha, \beta}\left(\frac{H^{2} \cdot \operatorname{ch}_{1}(E)}{H^{3}}, \frac{2 H \cdot \operatorname{ch}_{2}(E)}{H^{3}}, \frac{3 \operatorname{ch}_{3}(E)}{H^{3}}\right) .
$$

In particular, $Q_{\alpha, \beta}(E)=0$ describes a numerical wall in tilt stability.

\subsection{Further properties}

We will need the following modification of [CH16, Proposition 8.3]. It is a highly convenient tool to control the rank of destabilizing subobjects.

Lemma 2.4. Assume that a tilt semistable object $E$ is destabilized by either a subobject $F \hookrightarrow E$ or a quotient $E \rightarrow F$ in $\mathrm{Coh}^{\beta}(X)$ inducing a non-empty semicircular wall $W$. Assume further that we have $\operatorname{ch}_{0}(F)>\operatorname{ch}_{0}(E) \geqslant 0$. Then the inequality

$$
\rho_{W}^{2} \leqslant \frac{\Delta(E)}{4 \operatorname{ch}_{0}(F)\left(\operatorname{ch}_{0}(F)-\operatorname{ch}_{0}(E)\right)}
$$

holds.

Proof. For all $(\alpha, \beta) \in W$, we have the inequalities $H^{2} \cdot \operatorname{ch}_{1}^{\beta}(E) \geqslant H^{2} \cdot \operatorname{ch}_{1}^{\beta}(F) \geqslant 0$. This can be rewritten as

$$
H^{2} \cdot \operatorname{ch}_{1}(E)+\beta\left(H^{3} \cdot \operatorname{ch}_{0}(F)-H^{3} \cdot \operatorname{ch}_{0}(E)\right) \geqslant H^{2} \cdot \operatorname{ch}_{1}(F) \geqslant \beta H^{3} \cdot \operatorname{ch}_{0}(F) .
$$

Since $H^{2} \cdot \operatorname{ch}_{1}(F)$ is independent of $\beta$, we can maximize the right-hand side and minimize the lefthand side individually in the full range of $\beta$ between $s_{W}-\rho_{W}$ and $s_{W}+\rho_{W}$. By our assumptions, this leads to

$$
H^{2} \cdot \operatorname{ch}_{1}(E)+\left(s_{W}-\rho_{W}\right)\left(H^{3} \cdot \operatorname{ch}_{0}(F)-H^{3} \cdot \operatorname{ch}_{0}(E)\right) \geqslant\left(s_{W}+\rho_{W}\right) H^{3} \cdot \operatorname{ch}_{0}(F) .
$$

By rearranging the terms and squaring, we get

$$
\begin{aligned}
\left(2 H^{3} \cdot \operatorname{ch}_{0}(F)-H^{3} \cdot \operatorname{ch}_{0}(E)\right)^{2} \rho_{W}^{2} & \leqslant\left(H^{2} \cdot \operatorname{ch}_{1}(E)-H^{3} \cdot \operatorname{ch}_{0}(E) s_{W}\right)^{2} \\
& =\left(H^{3} \cdot \operatorname{ch}_{0}(E)\right)^{2} \rho_{W}^{2}+\left(H^{3}\right)^{2} \Delta(E) .
\end{aligned}
$$




\section{Derived CATEgories AND THE Genus of SPACE CURVES}

The claim follows by simply solving for $\rho_{W}^{2}$.

Objects that are stable for $\alpha \gg 0$ are closely related to slope semistable objects.

Lemma 2.5 ([BMS16, Lemma 2.7]). If $E \in \mathrm{Coh}^{\beta}(X)$ is $\nu_{\alpha, \beta}$-semistable for all $\alpha \gg 0$, then it satisfies one of the following conditions:

(i) we have $\mathcal{H}^{-1}(E)=0$, and $\mathcal{H}^{0}(E)$ is a torsion-free slope semistable sheaf;

(ii) we have $\mathcal{H}^{-1}(E)=0$, and $\mathcal{H}^{0}(E)$ is a torsion sheaf; or

(iii) the sheaf $\mathcal{H}^{-1}(E)$ is torsion-free slope semistable, and $\mathcal{H}^{0}(E)$ is either 0 or a torsion sheaf supported in dimension less than or equal to one.

Conversely, assume that $E \in \operatorname{Coh}(X)$ is a torsion-free slope stable sheaf and $\beta<\mu(E)$. Then $E \in \mathrm{Coh}^{\beta}(X)$ is $\nu_{\alpha, \beta}$-stable for $\alpha \gg 0$.

Instead of directly using the usual derived dual, we define

$$
\mathbb{D}: \quad \mathrm{D}^{\mathrm{b}}\left(\mathbb{P}^{3}\right) \rightarrow \mathrm{D}^{\mathrm{b}}\left(\mathbb{P}^{3}\right), \quad E \mapsto \mathbf{R} \mathcal{H o m}(E, \mathcal{O})[1] .
$$

Proposition 2.6 ([BMT14, Proposition 5.1.3]). Assume that $E \in \mathrm{Coh}^{\beta}(X)$ is $\nu_{\alpha, \beta}$-semistable with $\nu_{\alpha, \beta}(E) \neq \infty$. Then there exists a triangle

$$
\tilde{E} \rightarrow \mathbb{D}(E) \rightarrow T[-1] \rightarrow \tilde{E}[1],
$$

where $\tilde{E} \in \mathrm{Coh}^{-\beta}(X)$ is $\nu_{\alpha,-\beta}$-semistable and $T$ is a torsion sheaf supported in dimension zero.

Finally, the following elementary result will be used several times.

Lemma 2.7. Let $\beta_{0}=p / q \in \mathbb{Q}$, with $p, q$ coprime. Let $E \in \operatorname{Coh}^{\beta_{0}}(X)$ be such that either $\operatorname{ch}_{1}^{\beta_{0}}(E)=(1 / q) \cdot H$ or $\operatorname{ch}_{1}^{\beta_{0}}(E)=0$. Then, $E$ does not have any wall on the ray $\beta=\beta_{0}$ unless it is the unique vertical wall. More precisely, for all $\alpha_{1}, \alpha_{2}>0$, the object $E$ is $\nu_{\alpha_{1}, \beta_{0}}$-(semi)stable if and only if it is $\nu_{\alpha_{2}, \beta_{0}}$-(semi) stable.

\section{Classical bounds beyond projective space}

The main goal of this section is to prove Theorem 3.1 below. Let $X$ be a smooth projective variety over an algebraically closed field $\mathbb{F}$ for which Assumptions A, B, and $\mathrm{C}$ hold. The examples to keep in mind for $X$ are $\mathbb{P}^{3}$ and principally polarized abelian threefolds in characteristic zero. We denote by $H$ the ample generator of $\mathrm{NS}(X)$, for example, the hyperplane class for $\mathbb{P}^{3}$ or a $\Theta$-divisor for an abelian threefold.

The degree of a hypersurface $Y \subset X$ is defined as $k=\left(H^{2} \cdot Y\right) / H^{3}$. If $C \subset X$ is a onedimensional closed subscheme, we define its degree as $d=(H \cdot C) / H^{3}$. Note that $k \in \mathbb{Z}$ and $d \in \frac{1}{2} \mathbb{Z}$. If $X=\mathbb{P}^{3}$, we even have $d \in \mathbb{Z}$. The arithmetic genus of $C$ is defined as $g=1-\chi\left(\mathcal{O}_{C}\right)$. By the Hirzebruch-Riemann-Roch theorem, we know

$$
g=1-\chi\left(\mathcal{O}_{C}\right)=1+\frac{K_{X} \cdot C}{2}-\operatorname{ch}_{3}\left(\mathcal{O}_{C}\right)=1+\frac{K_{X} \cdot C}{2}+\operatorname{ch}_{3}\left(\mathcal{I}_{C}\right) .
$$

Therefore, bounding $g$ is equivalent to bounding $\operatorname{ch}_{3}\left(\mathcal{I}_{C}\right)$. The following error terms for any $d \in \frac{1}{2} \mathbb{Z}$ will occur in this and subsequent statements:

$$
\varepsilon(d, 1)= \begin{cases}1 / 24 & \text { if } d \notin \mathbb{Z}, \\ 0 & \text { if } d \in \mathbb{Z},\end{cases}
$$




\section{E. MACRÌ AND B. SCHMIDT}

and for $k>1$

$$
\begin{aligned}
\tilde{\varepsilon}(d, k) & =\frac{1}{2} f\left(k-f-1+\frac{f}{k}\right), \\
\varepsilon(d, k) & =\tilde{\varepsilon}(d, k)+\varepsilon(d, 1),
\end{aligned}
$$

where $d \equiv-f(\bmod k)$ and $0 \leqslant f<k$. The inclusion of $\varepsilon(d, 1)$ is related to the fact that Assumption A says that $\operatorname{ch}_{3}(E) \in \frac{1}{6} H^{3}$ for any object $E \in \mathrm{D}^{\mathrm{b}}(X)$. It simply constitutes a rounding term; all statements can be equivalently stated with $\tilde{\varepsilon}(d, k)$, and we will do so throughout the proofs for simplification.

Theorem 3.1. Let $k \in \mathbb{Z}_{>0}$ and $d \in \frac{1}{2} \mathbb{Z}_{>0}$, and let $C \subset X$ be an integral curve of degree $d$. Further, assume

- $H^{0}\left(X, I_{C}\left((k-1) H^{\prime}\right)=0\right.$ for any divisor $H^{\prime}$ in the same numerical class as $H$, and

- $d>k(k-1)$.

Then

$$
\frac{\operatorname{ch}_{3}\left(\mathcal{I}_{C}\right)}{H^{3}} \leqslant E(d, k):=\frac{d^{2}}{2 k}+\frac{d k}{2}-\varepsilon(d, k)
$$

We will also write

$$
\tilde{E}(d, k)=\frac{d^{2}}{2 k}+\frac{d k}{2}-\tilde{\varepsilon}(d, k)
$$

\subsection{Stable rank one objects}

Recall that for any curve $C \subset \mathbb{P}^{3}$ of degree $d$ and genus $g$, the inequality

$$
g \leqslant \frac{1}{2}(d-1)(d-2)
$$

holds. We will prove the following generalization to tilt semistable objects on $X$ whose Chern character is that of an ideal sheaf. This corresponds to the $k=1$ version of Theorem 3.1.

Proposition 3.2. Let $E \in \operatorname{Coh}^{\beta}(X)$ be a $\nu_{\alpha, \beta}$-semistable object for some $(\alpha, \beta)$ with either $H \cdot \operatorname{ch}(E)=(1,0,-d, e)$ or $H \cdot \operatorname{ch}(E)=(-1,0, d, e)$. Then

$$
e \leqslant \frac{1}{2} d(d+1)-\varepsilon(d, 1)=E(d, 1) .
$$

Note that the condition $\operatorname{ch}_{1}(E)=0$ is no real restriction since it can always be achieved by tensoring with an appropriate line bundle. In order to prove the proposition, we will first need to deal with torsion sheaves supported on a hypersurface of class $H$. The following lemma generalizes [Sch15, Lemma 5.4] from $\mathbb{P}^{3}$ to $X$.

Lemma 3.3. Let $E \in \operatorname{Coh}^{\beta}(X)$ be a $\nu_{\alpha, \beta}$-semistable object with $H \cdot \operatorname{ch}(E)=(0,1, d, e)$. Then

$$
e \leqslant \frac{1}{24}+\frac{1}{2} d^{2}-\varepsilon\left(d+\frac{1}{2}, 1\right) .
$$

Proof. As previously, the term $\varepsilon\left(d+\frac{1}{2}, 1\right)$ is simply a rounding term, and it is enough to show that $e \leqslant \frac{1}{24}+\frac{1}{2} d^{2}$. For any $(\alpha, \beta)$ in the semidisk $\alpha^{2}+(\beta-d)^{2} \leqslant \frac{1}{4}$, the inequality $Q_{\alpha, \beta}(E) \geqslant 0$ implies the claim. We are done if we can show that there is no wall outside this semidisk. Lemma 2.4 implies that if a wall has radius squared larger than $\frac{1}{4} \Delta(E)=\frac{1}{4}$, it must be induced by a rank zero subobject. But such a subobject destabilizes $E$ either for all $(\alpha, \beta)$ or for none.

Proof of Proposition 3.2. Assume $\operatorname{ch}_{0}(E)=-1$. By Proposition 2.6, there exists a triangle

$$
\tilde{E} \rightarrow \mathbb{D}(E) \rightarrow T[-1] \rightarrow \tilde{E}[1],
$$




\section{Derived CATEGories AND THE GENus of SPACE CURVES}

where $\tilde{E} \in \mathrm{Coh}^{-\beta(X)}$ is $\nu_{\alpha,-\beta}$-semistable and $T$ is a torsion sheaf supported in dimension zero. We have $\operatorname{ch}(\tilde{E})=\left(1,0,-\operatorname{ch}_{2}(E), \operatorname{ch}_{3}(E)+\operatorname{ch}_{3}(T)\right)$. Thus, it is enough to deal with the $\operatorname{ch}_{0}(E)=1$ case. The proof of that case is by induction on $d$. If $d=0$, then $Q_{\alpha, \beta}(E) \geqslant 0$ is equivalent to $e \leqslant 0$. Let $d=\frac{1}{2}$. Then we have $\operatorname{ch}^{-1}(E)=\left(1,1,0, e-\frac{1}{3}\right)$. All semicircular walls intersect the vertical line $\beta=-1$. Since there is no vertical wall at $\beta=-1$, this implies that $E$ has to be stable for $\beta=-1$ regardless of $\alpha$. Therefore, we can use $Q_{0,-1}(E) \geqslant 0$ to obtain $e \leqslant \frac{1}{3}$. Let $d \geqslant 1$, and assume for a contradiction that

$$
e>\frac{1}{2} d(d+1) \text {. }
$$

Note again that we can ignore $\varepsilon(d, 1)$ because it is just a rounding term. We write $s_{Q}:=-3 e / 2 d$. Assumption C implies

$$
\alpha^{2}+\left(\beta-s_{Q}\right)^{2} \geqslant \frac{9 e^{2}-8 d^{3}}{4 d^{2}}=: \rho_{Q}^{2} .
$$

This equation describes the complement of a semidisk, and there are no stable objects inside. Therefore, a potentially stable object must become strictly semistable at some larger semicircular wall. We will show that $E$ can only be destabilized by either a subobject $F \hookrightarrow E$ or a quotient $E \rightarrow F$ with $\operatorname{ch}_{0}(F)=1$. Let $\operatorname{ch}_{0}(F)=r \geqslant 1$ and $H^{2} \cdot \operatorname{ch}_{1}(F)=x H^{3}$. We have

$$
\rho_{Q}^{2}-\frac{d}{4}=\frac{9 e^{2}-8 d^{3}}{4 d^{2}}-\frac{d}{4}>\frac{9}{16}(d-1)^{2} \geqslant 0
$$

and can deduce $r=1$ from Lemma 2.4 .

The center of the numerical wall between $E$ and $\mathcal{O}(-2 H)$ is given by $s(E, \mathcal{O}(-2 H))=-\frac{1}{2} d-1$. This compares to $s_{Q}$ as follows:

$$
s(E, \mathcal{O}(-2 H))-s_{Q}>\frac{1}{4}(d-1) \geqslant 0 .
$$

Therefore, all walls are bigger than the numerical wall with $\mathcal{O}(-2 H)$; that is, $x=-1$. To simplify the notation, we will write

$$
H \cdot \operatorname{ch}(F)=(1,0,-y, z) \cdot(H \cdot \operatorname{ch}(\mathcal{O}(-H)))=\left(1,-1,-y+\frac{1}{2}, y+z-\frac{1}{6}\right) .
$$

The center of the wall is given by $s(E, F)=y-d-\frac{1}{2}$. In order for the wall to be outside the semidisk with no semistable objects, the inequality

$$
y-d-\frac{1}{2} \leqslant-\frac{3 e}{2 d}<-\frac{3}{4}(d+1)
$$

needs to hold. This implies

$$
0 \leqslant y<\frac{1}{4}(d-1) \leqslant \frac{1}{2}(d-1) .
$$

By induction, we know $z \leqslant y(y+1) / 2$. Let $G$ be the quotient $E / F$, respectively the subobject of the map $E \rightarrow F$. We have

$$
H \cdot \operatorname{ch}(G)=\left(0,1, y-d-\frac{1}{2}, e-z-y+\frac{1}{6}\right) .
$$

Lemma 3.3 implies

$$
\begin{aligned}
0 & \leqslant \frac{1}{24}+\frac{(y-d-1 / 2)^{2}}{2}-\left(e-z-y+\frac{1}{6}\right)=\frac{(d-y)^{2}}{2}+\frac{d+y}{2}+z-e \\
& <\frac{(d-y)^{2}}{2}+\frac{d+y}{2}+\frac{y(y+1)}{2}-\frac{d(d+1)}{2}=y^{2}+y-d y \leqslant 0 .
\end{aligned}
$$

The final inequality is obtained as follows: in $y$, the parabola $y^{2}+y-d y$ has a minimum for $y_{0}=\frac{1}{2}(d-1)>y$. Hence, the maximum occurs for $y=0$. 


\section{E. MACRÌ AND B. SCHMIDT}

\subsection{Stable rank zero objects}

In this section, we will prove the following bound for rank zero objects. It is a generalization of Lemma 3.3 beyond objects supported on divisors numerically equivalent to $H$.

Theorem 3.4. Let $E \in \operatorname{Coh}^{\beta}(X)$ be a $\nu_{\alpha, \beta}$-semistable object with $H \cdot \operatorname{ch}(E)=(0, c, d, e)$, where $c>0$. Then

$$
e \leqslant \frac{c^{3}}{24}+\frac{d^{2}}{2 c}-\varepsilon\left(d+\frac{c^{2}}{2}, c\right)
$$

As in the previous section, we will replace $\varepsilon$ by $\tilde{\varepsilon}$ and $E$ by $\tilde{E}$ in the proof since the difference is just a rounding term that comes for free at the end. The case $c=1$ was already shown in Lemma 3.3. Throughout this section, we will prove the theorem, assuming its notation and $c \geqslant 2$.

Lemma 3.5. Assume that (3.1) does not hold.

(i) We can bound $\tilde{\varepsilon}\left(d+\frac{1}{2} c^{2}, c\right) \leqslant \frac{1}{8}\left(c^{2}-c\right)$.

(ii) The radius $\rho_{Q}$ of the semidisk $Q_{\alpha, \beta}(E) \leqslant 0$ is given by

$$
\rho_{Q}^{2}=\frac{6 c e-3 d^{2}}{c^{2}}>\frac{1}{4} c^{2}-\frac{6 \tilde{\varepsilon}\left(d+\frac{1}{2} c^{2}, c\right)}{c} \geqslant \frac{c^{2}-3 c+3}{4}>\left(\frac{2 c-3}{4}\right)^{2} .
$$

(iii) The object $E$ is destabilized along a wall $W$ induced by $0 \rightarrow F \rightarrow E \rightarrow G \rightarrow 0$ or $0 \rightarrow G \rightarrow E \rightarrow F \rightarrow 0$, where $F$ has positive rank. Let $H \cdot \operatorname{ch}(F)=(r, x, y, z)$.

(iv) We have $r=1$.

(v) There are inequalities

$$
\frac{c^{2}}{8}+\frac{d x}{c}-\frac{d^{2}}{2 c^{2}}-\frac{3 f}{2}+\frac{3 f^{2}}{2 c}+\frac{3 f}{2 c}-\frac{3 f^{2}}{2 c^{2}}<y \leqslant \min \left\{\frac{x^{2}}{2}, \frac{(c-x)^{2}}{2}+d\right\} .
$$

(vi) The inequalities

$$
\frac{d}{c}+\frac{c}{2}+\frac{3}{4} \geqslant x \geqslant \frac{d}{c}+\frac{c}{2}-\frac{3}{4}
$$

hold; that is, either $x=d / c+c / 2+f / c$, or $x=d / c+c / 2+f / c-1$.

(vii) If $c=2$, then $y=\min \left\{\frac{1}{2} x^{2}, \frac{1}{2}(2-x)^{2}+d\right\}$.

(viii) We have

$$
z \leqslant \frac{x^{4}}{8}-\frac{x^{3}}{3}-\frac{x^{2} y}{2}+\frac{x^{2}}{4}+x y+\frac{y^{2}}{2}-\frac{y}{2}
$$

(ix) We have

$$
\begin{aligned}
e \leqslant & \frac{c^{4}}{8}-\frac{c^{3} x}{2}+\frac{3 c^{2} x^{2}}{4}-\frac{c x^{3}}{2}+\frac{x^{4}}{4}-\frac{c^{3}}{3}+\frac{c^{2} d}{2}+c^{2} x-c d x-c x^{2}+\frac{d x^{2}}{2}-\frac{c^{2} y}{2} \\
& +c x y-x^{2} y+\frac{c^{2}}{4}-c d+\frac{d^{2}}{2}-\frac{c x}{2}+d x+\frac{x^{2}}{2}+c y-d y+y^{2}+\frac{d}{2}-y .
\end{aligned}
$$

Proof. (i) The function $\tilde{\varepsilon}\left(d+\frac{1}{2} c^{2}, c\right)$ has a maximum for $f=\frac{1}{2} c$, and in that case, $\tilde{\varepsilon}\left(d+\frac{1}{2} c^{2}, c\right)=$ $\frac{1}{8}\left(c^{2}-c\right)$.

(ii) The semidisk $Q_{\alpha, \beta}(E) \leqslant 0$ is given by

$$
\alpha^{2}+\left(\beta-\frac{d}{c}\right)^{2} \leqslant \frac{6 e-3 d^{2}}{c^{2}} .
$$




\section{Derived CATEGories AND the Genus of SPACE CURVES}

The fact that (3.1) does not hold and part (i) lead to the inequalities

$$
\frac{6 c e-3 d^{2}}{c^{2}}>\frac{c^{2}}{4}-\frac{6 \tilde{\varepsilon}\left(d+\frac{1}{2} c^{2}, c\right)}{c} \geqslant \frac{c^{2}-3 c+3}{4}>\left(\frac{2 c-3}{4}\right)^{2} .
$$

(iii) By part (ii), the region $Q_{\alpha, \beta}(E)<0$ is non-empty and there has to be a wall for $E$.

(iv) When $E$ gets destabilized, there is either a subobject or a quotient $F$ with non-negative rank. If $\operatorname{ch}_{0}(F) \geqslant 2$, then by Lemma 2.4 , the radius $\rho_{W}$ must satisfy

$$
\rho_{W}^{2} \leqslant \frac{\Delta(E)}{4 \operatorname{ch}_{0}(F)^{2}} \leqslant \frac{c^{2}}{16} \leqslant \frac{c^{2}}{4}-\frac{3 c}{4}+\frac{3}{4} .
$$

But by part (ii), the wall $W$ is too small to exist. Assume $\operatorname{ch}_{0}(F)=0$. Then the equation $\nu_{\alpha, \beta}(F)=\nu_{\alpha, \beta}(E)$ is independent of $(\alpha, \beta)$ and cannot induce a wall.

(v) Solving the two inequalities $\Delta(F), \Delta(G) \geqslant 0$ for y leads to

$$
y \leqslant \min \left\{\frac{x^{2}}{2}, \frac{(c-x)^{2}}{2}+d\right\} .
$$

We know that $W$ must be outside the semidisk $Q_{\alpha, \beta}(E)<0$. The radius of $W$ can be computed as

$$
\rho_{W}^{2}=\frac{2 c^{2} y+d^{2}-2 c d x}{c^{2}} \geqslant \rho_{Q}^{2} \geqslant \frac{c^{2}}{4}-\frac{6 \tilde{\varepsilon}\left(d+\frac{1}{2} c^{2}, c\right)}{c} .
$$

Solving the inequality for $y$ leads to the claimed lower bound for $y$.

(vi) We know that $\rho_{W} \geqslant(2 c-3) / 4$ and $s_{W}=d / c$. By the construction of $\operatorname{Coh}^{\beta}(X)$, we have $0 \leqslant\left(H^{2} \cdot \operatorname{ch}_{1}^{\beta}(F)\right) / H^{3} \leqslant c$ for all $\beta$ appearing along the wall $W$. Rearranging the terms leads to

$$
c+\beta \geqslant x \geqslant \beta \text {. }
$$

Since the middle term is independent of $\beta$, we can vary $\beta$ independently on the left and right to get

$$
\frac{d}{c}+\frac{c}{2}+\frac{3}{4} \geqslant c+s_{W}-\rho_{W} \geqslant x \geqslant s_{W}+\rho_{W} \geqslant \frac{d}{c}+\frac{c}{2}-\frac{3}{4} .
$$

(vii) Assume $c=2$. If $x=d / c+c / 2+f / c$, then

$$
\frac{(c-x)^{2}}{2}+d-\left(\frac{c^{2}}{8}+\frac{d x}{c}-\frac{d^{2}}{2 c^{2}}-\frac{3 f}{2}+\frac{3 f^{2}}{2 c}+\frac{3 f}{2 c}-\frac{3 f^{2}}{2 c^{2}}\right)=-\frac{f(f-1)}{4}<\frac{1}{2} .
$$

If $x=d / c+c / 2+f / c-1$, then

$$
\frac{x^{2}}{2}-\left(\frac{c^{2}}{8}+\frac{d x}{c}-\frac{d^{2}}{2 c^{2}}-\frac{3 f}{2}+\frac{3 f^{2}}{2 c}+\frac{3 f}{2 c}-\frac{3 f^{2}}{2 c^{2}}\right)=-\frac{(f-1)(f-2)}{4}<\frac{1}{2} .
$$

In both cases, we can conclude by part (v).

(viii) Since $F$ has rank 1, we can use Proposition 3.2 to bound $z$ as claimed.

(ix) Since $G$ has rank -1, Proposition 3.2 applies, and together with (viii) the last claim follows.

Proof of Theorem 3.4. We need to maximize the function

$$
\begin{aligned}
g_{c, d, x}(y):= & \frac{c^{4}}{8}-\frac{c^{3} x}{2}+\frac{3 c^{2} x^{2}}{4}-\frac{c x^{3}}{2}+\frac{x^{4}}{4}-\frac{c^{3}}{3}+\frac{c^{2} d}{2}+c^{2} x-c d x-c x^{2}+\frac{d x^{2}}{2}-\frac{c^{2} y}{2} \\
& +c x y-x^{2} y+\frac{c^{2}}{4}-c d+\frac{d^{2}}{2}-\frac{c x}{2}+d x+\frac{x^{2}}{2}+c y-d y+y^{2}+\frac{d}{2}-y
\end{aligned}
$$




\section{E. MACRÌ AND B. SCHMIDT}

under the numerical constraints imposed on $c, d, x, y$ by Lemma 3.5. The argument works as follows: We will show that $g_{c, d, x}(y)$ is increasing in $y$ and that we can, therefore, reduce to

$$
y=\min \left\{\frac{x^{2}}{2}, \frac{(c-x)^{2}}{2}+d\right\} .
$$

Note that we can assume $d \geqslant 3$ since for $d=2$, we established that this is the only possible value for $y$. The function $g_{c, d, x}(y)$ is a parabola in $y$ with minimum at

$$
y_{0}=\frac{c^{2}}{4}-\frac{c x}{2}+\frac{x^{2}}{2}-\frac{c}{2}+\frac{d}{2}+\frac{1}{2} .
$$

The proof will proceed individually for each of the two possible values for $x$.

(1) Assume $x=d / c+c / 2+f / c$. Then

$$
\begin{aligned}
y-y_{0} & >\left(\frac{c^{2}}{8}+\frac{d x}{c}-\frac{d^{2}}{2 c^{2}}-\frac{3 f}{2}+\frac{3 f^{2}}{2 c}+\frac{3 f}{2 c}-\frac{3 f^{2}}{2 c^{2}}\right)-\left(\frac{c^{2}}{4}-\frac{c x}{2}+\frac{x^{2}}{2}-\frac{c}{2}+\frac{d}{2}+\frac{1}{2}\right) \\
& =\frac{c}{2}-\frac{3 f}{2}+\frac{3 f^{2}}{2 c}+\frac{3 f}{2 c}-\frac{2 f^{2}}{c^{2}}-\frac{1}{2}=: h_{c}(f) .
\end{aligned}
$$

Note that $h_{c}(f)$ is a parabola in $f$ with minimum at $f_{0}=\left(3 c^{2}-3 c\right) /(6 c-8)$. For $c \geqslant 3$, we get

$$
h_{c}\left(f_{0}\right)=\frac{(3 c-7)(c-1)}{24 c-32}>0
$$

and thus, $g_{c, d, x}(y)$ is increasing under our restrictions on $c, d, x, y$. Finally

$$
g_{c, d, x}\left(\frac{(c-x)^{2}}{2}+d\right)=\frac{c^{3}}{24}+\frac{d^{2}}{2 c}-\tilde{\varepsilon}\left(d+\frac{c^{2}}{2}, c\right) .
$$

(2) Assume $x=d / c+c / 2+f / c-1$. Then

$$
\begin{aligned}
y-y_{0} & >\left(\frac{c^{2}}{8}+\frac{d x}{c}-\frac{d^{2}}{2 c^{2}}-\frac{3 f}{2}+\frac{3 f^{2}}{2 c}+\frac{3 f}{2 c}-\frac{3 f^{2}}{2 c^{2}}\right)-\left(\frac{c^{2}}{4}-\frac{c x}{2}+\frac{x^{2}}{2}-\frac{c}{2}+\frac{d}{2}+\frac{1}{2}\right) \\
& =\frac{c}{2}-\frac{3 f}{2}+\frac{3 f^{2}}{2 c}+\frac{5 f}{2 c}-\frac{2 f^{2}}{c^{2}}-1=: h_{c}(f) .
\end{aligned}
$$

Note that $h_{c}(f)$ is a parabola in $f$ with minimum at $f_{0}=\left(3 c^{2}-5 c\right) /(6 c-8)$. For $c \geqslant 3$, we get

$$
h_{c}\left(f_{0}\right)=\frac{(3 c-7)(c-1)}{24 c-32}>0
$$

and thus, $g_{c, d, x}(y)$ is increasing under our restrictions on $c, d, x, y$. Finally,

$$
g_{c, d, x}\left(\frac{x^{2}}{2}\right)=\frac{c^{3}}{24}+\frac{d^{2}}{2 c}-\tilde{\varepsilon}\left(d+\frac{c^{2}}{2}, c\right) .
$$

\subsection{Stable rank two objects}

In this section, we prove the following bound for tilt stable rank two objects.

Theorem 3.6. Let $E \in \operatorname{Coh}(X)$ be a $\nu_{\alpha, \beta}$-semistable rank two object for some $(\alpha, \beta)$ with $H \cdot \operatorname{ch}(E)=(2, c, d, e)$.

(i) If $c=-1$, then $d \leqslant 0$ and $e \leqslant \frac{1}{2} d^{2}-d+\frac{5}{24}-\varepsilon\left(d+\frac{1}{2}, 1\right)$.

(ii) If $c=0$, then $d \leqslant 0$.

(a) If $d=0$, then $e \leqslant 0$. 


\section{DERIVED CATEGORIES AND THE GENUS OF SPACE CURVES}

(b) If $d=-\frac{1}{2}$, then $e \leqslant \frac{1}{6}$.

(c) If $d \leqslant-1$, then $e \leqslant \frac{1}{2} d^{2}+\frac{5}{24}-\varepsilon\left(d+\frac{1}{2}, 1\right)$.

Note that

$$
\frac{d^{2}}{2}+\frac{5}{24}-\varepsilon\left(d+\frac{1}{2}, 1\right) \in \frac{1}{6} \mathbb{Z}
$$

for any $d \in \frac{1}{2} \mathbb{Z}$. Again, we will be able to ignore $\varepsilon\left(d+\frac{1}{2}, 1\right)$ as it is simply a rounding term.

The bounds on $d$ are a consequence of the Bogomolov inequality. The bounds on $e$ will be proved via an induction on the discriminant $\Delta(E)$. We start with two lemmas for cases of low discriminant.

Lemma 3.7. If $E \in \operatorname{Coh}^{\beta}(X)$ is a tilt semistable object with $H \cdot \operatorname{ch}(E)=(2,0,0, e)$, then $e \leqslant 0$. Proof. Since $E \in \operatorname{Coh}^{\beta}(X)$, we must have $\beta<0$. The inequality on $e$ is then equivalent to $Q_{\alpha, \beta}(E) \geqslant 0$.

Lemma 3.8. If $E \in \operatorname{Coh}^{\beta}(X)$ is a tilt semistable object with $H \cdot \operatorname{ch}(E)=\left(2,0,-\frac{1}{2}, e\right)$, then $e \leqslant \frac{1}{6}$.

Proof. If $E$ is semistable for some $\alpha>0$ and $\beta \in \mathbb{R}$ inside the closed semidisk $\alpha^{2}+\left(\beta+\frac{3}{4}\right)^{2} \leqslant \frac{1}{16}$, then $Q_{\alpha, \beta}(E) \geqslant 0$ implies $e \leqslant \frac{1}{4}$. By Assumption A, this means $e \leqslant \frac{1}{6}$.

Next, we will show that $E$ has to be semistable for some point inside this closed semidisk. If not, then $E$ is destabilized by a semistable subobject $F$ along the vertical line $\beta=-1$. If $\operatorname{ch}_{0}(F) \leqslant 0$, then we work with the quotient $E / F$ instead. Therefore, we can assume $\operatorname{ch}_{0}(F) \geqslant 1$. We can compute

$$
H \cdot \operatorname{ch}_{\leqslant 2}^{-1}(E)=\left(2,2, \frac{1}{2}\right) .
$$

Let $H \cdot \operatorname{ch}_{\leqslant 2}^{-1}(F)=(r, x, y)$. Then the definition of $\operatorname{Coh}^{\beta}(X)$ and the fact that we are not dealing with a vertical wall implies $0<x<2$; that is, $x=1$. At the wall, we have

$$
y-\frac{1}{2} r \alpha^{2}=\nu_{\alpha,-1}(F)=\nu_{\alpha,-1}(E)=\frac{1}{4}-\frac{1}{2} \alpha^{2} .
$$

If $r=1$, then this implies $y=\frac{1}{4}$, in contradiction to Assumption A. If $r \geqslant 2$, we get $y>\frac{1}{4}$. However, that implies $\Delta(F)=1-2 r y \leqslant 1-4 y<0$, giving a contradiction.

Lemma 3.9. Let $E \in \operatorname{Coh}^{\beta}(X)$ be a tilt semistable object with $H \cdot \operatorname{ch}(E)=(2, c, d, e)$. Assume either

(i) $c=-1, d \leqslant 0$, and $e \geqslant \frac{1}{2} d^{2}-d+\frac{1}{3}$; or

(ii) $c=0, d \leqslant-1$, and $e \geqslant \frac{1}{2} d^{2}+\frac{1}{3}$.

Then $E$ is destabilized along a semicircular wall induced by an exact sequence $0 \rightarrow F \rightarrow E \rightarrow$ $G \rightarrow 0$, where $F$ and $G$ have rank at most two.

Proof. (i) Assume $c=-1$. Then the radius $\rho_{Q}$ of the semidisk $Q_{\alpha, \beta}(E) \leqslant 0$ satisfies

$$
\begin{aligned}
\rho_{Q}^{2}-\frac{\Delta(E)}{12} & =\frac{16 d^{3}-3 d^{2}+36 d e+36 e^{2}-6 e}{(4 d-1)^{2}}+\frac{d}{3}-\frac{1}{12} \\
& \geqslant \frac{108 d^{4}+40 d^{3}+24 d^{2}-60 d+23}{12(4 d-1)^{2}}>0 .
\end{aligned}
$$

By Lemma 2.4, any destabilizing subobjects or quotients must have rank less than or equal to two. 


\section{E. MACRÌ AND B. SCHMIDT}

(ii) Assume $c=0$. Then the radius $\rho_{Q}$ of the semidisk $Q_{\alpha, \beta}(E) \leqslant 0$ satisfies

$$
\begin{aligned}
\rho_{Q}^{2}-\frac{\Delta(E)}{12} & =\frac{4 d^{3}+9 e^{2}}{4 d^{2}}+\frac{d}{3} \\
& \geqslant \frac{27 d^{4}+64 d^{3}+36 d^{2}+12}{48 d^{2}}>0 .
\end{aligned}
$$

By Lemma 2.4, any destabilizing subobjects or quotients must have rank less than or equal to two.

Proof of Theorem 3.6. If $c=-1$, then $\Delta(E)=1-4 d \geqslant 0$. Since $d \in \frac{1}{2} \mathbb{Z}$, we get $d \leqslant 0$. If $c=0$, then $\Delta(E)=-4 d \geqslant 0$ implies $d \leqslant 0$. We will prove the bounds on $e$ simultaneously in both cases via induction on $\Delta(E)$. Lemmas 3.7 and 3.8 provide the start of the induction. To deduce a contradiction, assume that the upper bounds on $e$ claimed in the theorem do not hold.

The strategy of the proof is to show that there is no wall outside the semidisk $Q_{\alpha, \beta}(E)<0$ and, therefore, there is no wall for such an object. By Lemma 3.9, we know that $E$ is destabilized along a semicircular wall $W$ induced by a subobject $F \hookrightarrow E$ of rank less than or equal to two. By replacing $F$ with the quotient $E / F$ if necessary, we can assume that $F$ has rank one or two. Let $H \cdot \operatorname{ch}(F)=(r, x, y, z)$. Note that we have $\Delta(F)<\Delta(E)$, and we intend to use the induction hypothesis on $F$ in case $r=2$.

(1) Assume $c=-1, d \leqslant 0$, and $e \geqslant \frac{1}{2} d^{2}-d+\frac{1}{3}$.

(a) Assume that $F$ has rank one. Then

$$
Q_{0,-3 / 2}(E)=4 d^{2}-12 d-12 e+\frac{9}{4} \leqslant-2 d^{2}-\frac{7}{4}<0 .
$$

This implies

$$
x+\frac{3}{2}=\frac{H^{2} \cdot \operatorname{ch}_{1}^{-3 / 2}(F)}{H^{3}}>0 .
$$

Since $x \geqslant 0$ implies the wall to be on the wrong side of the vertical wall, we must have $x=-1$. The Bogomolov inequality $\Delta(F) \geqslant 0$ implies $y \leqslant \frac{1}{2}$. We need a second bound of $y$ from below. We have $s(E, F)=d-2 y$. Moreover, the center of the semidisk $Q_{\alpha, \beta}(E)<0$ is given by $s_{Q}=(d+6 e) /(4 d-1)$. Since no wall can be inside this semidisk, we must have

$$
0 \leqslant s_{Q}-s(E, F)=\frac{d+6 e}{4 d-1}-d+2 y \leqslant \frac{d^{2}-8 d y+4 d+2 y-2}{1-4 d} .
$$

This implies $y \geqslant\left(d^{2}+4 d-2\right) /(8 d-2)$. Applying Proposition 3.2 to $F$ gives $z \leqslant \frac{1}{2} y^{2}-2 y+\frac{17}{24}$. We can apply the same proposition to the quotient $E / F$ to obtain

$$
e \leqslant \frac{d^{2}}{2}-d y+\frac{y^{2}}{2}-\frac{d}{2}+\frac{y}{2}+z \leqslant \frac{d^{2}}{2}-d y+y^{2}-\frac{d}{2}-\frac{3 y}{2}+\frac{17}{24}=: \varphi_{d}(y) .
$$

We have to maximize this expression in $y$, which defines a parabola with minimum. Therefore, the maximum has to occur on the boundary. We can compute

$$
\varphi_{d}\left(\frac{1}{2}\right)=\frac{1}{2} d^{2}-d+\frac{5}{24} .
$$

Moreover,

$$
\frac{d^{2}}{2}-d+\frac{1}{3}-\varphi_{d}\left(\frac{d^{2}+4 d-2}{8 d-2}\right)=\frac{14 d^{4}+4 d^{3}+2 d^{2}-12 d+1}{8(4 d-1)^{2}}>0 .
$$




\section{Derived CATEgories AND THE Genus of SPACE CURVES}

(b) Assume that $F$ has rank two. As in the rank one case, we get

$$
x+3=\frac{H^{2} \cdot \operatorname{ch}_{1}^{-3 / 2}(F)}{H^{3}}>0 ;
$$

that is, $x \geqslant-2$. If $x=-1$, we are dealing with the vertical wall, and if $x \geqslant 0$, then the wall is on the wrong side of the vertical wall. Hence, we must have $x=-2$. The Bogomolov inequality says $y \leqslant 1$. We need to bound $y$ from below. We have $s(E, F)=d-y$. Moreover, the center of the semidisk $Q_{\alpha, \beta}(E)<0$ is given by $s_{Q}=(d+6 e) /(4 d-1)$. Since no wall can be inside this semidisk, we must have

$$
0 \leqslant s_{Q}-s(E, F)=\frac{d+6 e}{4 d-1}-d+y \leqslant \frac{d^{2}-4 d y+4 d+y-2}{1-4 d} .
$$

This implies $y \geqslant\left(d^{2}+4 d-2\right) /(4 d-1)$. In particular, for $d=0$, we have $1 \geqslant y \geqslant 2$, and such a wall simply cannot exist. Therefore, we can assume $d \leqslant-\frac{1}{2}$. Applying Lemma 3.3 to the quotient $E / F$ leads to

$$
e \leqslant \frac{1}{2}(d-y)^{2}+z+\frac{1}{24} .
$$

The next step is to apply induction to $F(1)$. If $y=1$, then $H \cdot \operatorname{ch}_{3}(F(1))=z+\frac{1}{3} \leqslant 0$; that is, $z \leqslant-\frac{1}{3}$. This leads to

$$
e \leqslant \frac{1}{2} d^{2}-d+\frac{5}{24}
$$

If $y=\frac{1}{2}$, then $\operatorname{ch}_{3}(F(1))=z-\frac{1}{6} \leqslant \frac{1}{6}$; that is, $z \leqslant \frac{1}{3}$. This leads to

$$
e \leqslant \frac{1}{2} d^{2}-\frac{1}{2} d+\frac{1}{2}<\frac{1}{2} d^{2}-d+\frac{1}{3} .
$$

Assume $y \leqslant 0$. Then we have

$$
\frac{d^{2}+4 d-2}{4 d-1} \leqslant y \leqslant 0
$$

This implies $d \leqslant-\frac{9}{2}$. We can apply induction to $F(1)$ to get $z \leqslant \frac{1}{2} y^{2}-2 y+\frac{11}{8}$. Therefore,

$$
e \leqslant \frac{1}{2} d^{2}-d y+y^{2}-2 y+\frac{17}{12}=: \varphi_{d}(y) \text {. }
$$

This expression defines a parabola with minimum in $y$. Therefore its maximum will occur on the boundary. We have

$$
\frac{1}{2} d^{2}-d+\frac{1}{3}-\varphi_{d}(0) \geqslant-d-\frac{13}{12}>0 .
$$

On the other boundary point, we have

$$
\frac{1}{2} d^{2}-d+\frac{1}{3}-\varphi_{d}\left(\frac{d^{2}+4 d-2}{4 d-1}\right)=\frac{36 d^{4}-12 d^{3}-40 d^{2}+20 d-13}{12(4 d-1)^{2}}>0 .
$$

(2) Assume $c=0, d \leqslant-1$, and $e \geqslant \frac{1}{2} d^{2}+\frac{1}{3}$.

(a) Assume that $F$ has rank one. Then

$$
Q_{0,-1}(E)=4 d^{2}-4 d-12 e \leqslant-2 d^{2}-4 d-4<0 .
$$

Thus, $W(F, E)$ must contain a point $(\alpha,-1)$, and we have

$$
0<\frac{H^{2} \cdot \mathrm{ch}_{1}^{-1}(F)}{H^{3}}=x+1 ;
$$

that is, $x>-1$. However, if $x \geqslant 0$, then we are dealing with either the vertical wall or a wall to the right of the vertical wall. Therefore, the wall cannot exist. 


\section{E. MACRÌ AND B. SCHMIDT}

(b) Assume that $F$ has rank two. Then as in the rank one case, we have $Q_{0,-1}(E)<0$, and thus,

$$
0<\frac{H^{2} \cdot \operatorname{ch}_{1}^{-1}(F)}{H^{3}}=x+2
$$

that is, $x>-2$. Again, $x \geqslant 0$ implies that we do not deal with a wall to the left of the vertical wall. Overall, we must have $x=-1$. The inequality $\Delta(F) \geqslant 0$ implies $y \leqslant 0$. We need to bound $y$ from below. We have $s(E, F)=d-y$. Moreover, the center of the semidisk $Q_{\alpha, \beta}(E)<0$ is given by $s_{Q}=3 e / 2 d$. Since no wall can be inside this semidisk, we must have

$$
0 \leqslant s_{Q}-s(E, F) \leqslant-\frac{d^{2}-4 d y-2}{4 d} .
$$

This implies $y \geqslant\left(d^{2}-2\right) / 4 d$. We can apply Lemma 3.3 to the quotient $E / F$ to get

$$
e \leqslant \frac{1}{2}(d-y)^{2}+z+\frac{1}{24} .
$$

The next step is to apply induction to $F$ to get $z \leqslant \frac{1}{2} y^{2}-y+\frac{5}{24}$, which implies

$$
e \leqslant \frac{1}{2}(d-y)^{2}+z+\frac{1}{24} \leqslant \frac{1}{2} d^{2}-d y+y^{2}-y+\frac{1}{4}=: \varphi_{d}(y) .
$$

This expression defines a parabola in $y$ with minimum. Therefore, the maximum will occur on a boundary point. We get $\frac{1}{2} d^{2}+\frac{1}{3}-\varphi_{d}(0) \geqslant \frac{1}{12}>0$. Moreover,

$$
\frac{1}{2} d^{2}+\frac{1}{3}-\varphi_{d}\left(\frac{d^{2}-2}{4 d}\right)=\frac{9 d^{4}+12 d^{3}-8 d^{2}-24 d-12}{48 d^{2}}>0 .
$$

\subsection{Bounding the arithmetic genus of integral curves}

We will now prove Theorem 3.1 in a series of lemmas. The proof will be by contradiction. We already dealt with the case $k=1$ in Proposition 3.2. Therefore, we will assume $k \geqslant 2$ in this proof.

Lemma 3.10. Under the assumptions of Theorem 3.1, the inequality $e>E(d, k)$ implies $e \geqslant$ $\tilde{E}(d, k)+\frac{1}{8}>\tilde{E}(d, k)$. In particular, Theorem 3.1 requires only to prove $e \leqslant \tilde{E}(d, k)$.

Proof. We know $e \in \frac{1}{6} \mathbb{Z}$. The statement now follows from the fact that $E(d, k) \in \frac{1}{6} \mathbb{Z}$ and $\varepsilon(d, 1)+\frac{1}{8} \leqslant \frac{1}{6}$.

LEMma 3.11. (i) The following bound holds: $\tilde{\varepsilon}(d, k) \leqslant \frac{1}{8} k^{2}-\frac{1}{8} k$.

(ii) If $h>k$ and $d>h(h-1)$, then $\tilde{E}(d, k)>\tilde{E}(d, h)$; that is, the function $\tilde{E}(d, k)$ is strictly decreasing in $k$ as long as $d>k(k-1)$.

Proof. The first part was already observed in Lemma 3.5 part (i); namely, $\tilde{\varepsilon}(d, k)$ is a parabola in $f$ with maximum at $f=\frac{1}{2} k$.

For the second part, it is enough to show $\tilde{E}(d, k)>\tilde{E}(d, k+1)$ whenever $d>k(k+1)$. Let $m \geqslant k+2$ be the unique integer such that $d=m k-f$, where $0 \leqslant f<k$. We will deal with the following three cases individually.

(1) Assume $m \geqslant 2 k+1$. Then $d>2 k^{2}$. We have

$$
\begin{aligned}
\tilde{E}(d, k)-\tilde{E}(d, k+1) & \geqslant\left(\frac{d^{2}}{2 k}+\frac{d k}{2}-\frac{k^{2}}{8}+\frac{k}{8}\right)-\left(\frac{d^{2}}{2(k+1)}+\frac{d(k+1)}{2}\right) \\
& =\frac{-k^{4}-4 d k^{2}+4 d^{2}-4 d k+k^{2}}{8\left(k^{2}+k\right)} .
\end{aligned}
$$




\section{DERIVED CATEGORIES AND THE GENUS OF SPACE CURVES}

Therefore, it is enough to show that the function

$$
\varphi_{k}(d)=-k^{4}-4 d k^{2}+4 d^{2}-4 d k+k^{2}
$$

is positive. The fact that $d>2 k^{2}$ implies $\varphi_{k}^{\prime}(d)=-4 k^{2}+8 d-4 k>0$. Thus, for $k \geqslant 2$, we have $\varphi_{k}(d) \geqslant \varphi_{k}\left(2 k^{2}\right)=7 k^{4}-8 k^{3}+k^{2}>0$. If $k=1$, then we have $d \geqslant k(k+1)+\frac{1}{2}=\frac{5}{2}$, and $\varphi_{1}\left(\frac{5}{2}\right)=5>0$.

(2) Assume $m \leqslant 2 k$ and $0 \leqslant f \leqslant 2 k-m+\frac{3}{2}$. Let $d \equiv-f^{\prime}(\bmod k+1)$ for $0 \leqslant f^{\prime}<k+1$. Then $f^{\prime}=m+f-k-1$ and $\tilde{E}(d, k)-\tilde{E}(d, k+1)=(k-f)(m-k-1)>0$.

(3) Assume $m \leqslant 2 k$ and $2 k-m+2 \leqslant f<k$. Note that this range for $f$ is non-empty if and only if $m \geqslant k+3$. Let $d \equiv-f^{\prime}(\bmod k+1)$ for $0 \leqslant f^{\prime}<k+1$. Then $f^{\prime}=m+f-2 k-2$, and $\tilde{E}(d, k)-\tilde{E}(d, k+1)=2 f k-3 k^{2}-f m+2 k m+f-3 k$ is linear in $f$ and increasing. Therefore, the minimum occurs at $f=2 k-m+2$, where

$$
\tilde{E}(d, k)-\tilde{E}(d, k+1)=(m-k-2)(m-k-1)>0 .
$$

Lemma 3.12. Let $C \subset X$ be an integral curve. Assume that $\mathcal{I}_{C}$ is destabilized via an exact sequence $0 \rightarrow E \rightarrow \mathcal{I}_{C} \rightarrow G \rightarrow 0$ in $\mathrm{Coh}^{\beta}(X)$ defining a semicircular wall in tilt stability. Then $E$ is a reflexive sheaf.

Proof. The long exact sequences

$$
0 \rightarrow \mathcal{H}^{-1}(E) \rightarrow 0 \rightarrow \mathcal{H}^{-1}(G) \rightarrow \mathcal{H}^{0}(E)=E \rightarrow \mathcal{I}_{C} \rightarrow \mathcal{H}^{0}(G) \rightarrow 0
$$

shows that $E$ is a sheaf. Since both $\mathcal{H}^{-1}(G)$ and $\mathcal{I}_{C}$ are torsion free sheaves, so is $E$. Let $Q$ be the cokernel of the natural inclusion $E \hookrightarrow E^{\vee \vee}$. If $Q=0$, we are done. If not, $Q$ has to be supported in dimension less than or equal to one. The strategy of the rest of the proof is to obtain a contradiction to $Q \neq 0$. We get a commutative diagram with exact sequences in $\operatorname{Coh}^{\beta}(X)$ as rows:

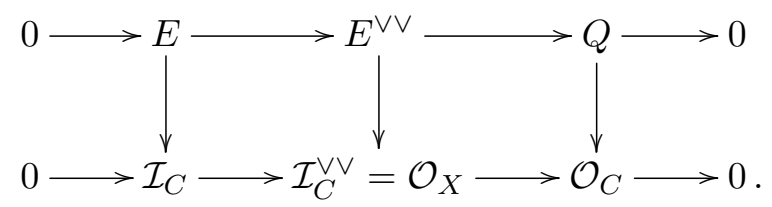

The kernel $K$ of $Q \rightarrow \mathcal{O}_{C}$ in $\operatorname{Coh}^{\beta}(X)$ is also a torsion sheaf supported in dimension less than or equal to one. The snake lemma leads to a map $K \rightarrow G$, but $G$ is semistable and this map has to be trivial. Therefore, we get an injection $K \hookrightarrow E^{\vee \vee}$. Since $E^{\vee \vee}$ is torsion free, we must have $K=0$. Because $C$ is integral, the injective map $Q \rightarrow \mathcal{O}_{C}$ implies that $Q$ is scheme-theoretically supported on $C$. Moreover, $E^{\vee \vee} \rightarrow \mathcal{O}_{X}$ is injective in $\operatorname{Coh}^{\beta}(X)$. Note that along the wall, we must have $0<H \cdot \operatorname{ch}_{1}^{\beta}(E)<H \cdot \operatorname{ch}_{1}^{\beta}\left(\mathcal{I}_{C}\right)=H \cdot \operatorname{ch}_{1}^{\beta}\left(\mathcal{O}_{X}\right)$. Let $d$ be the degree of $C$. The final contradiction is obtained via

$$
\begin{aligned}
\nu_{\alpha, \beta}\left(E^{\vee \vee}\right) & =\frac{d}{H \cdot \operatorname{ch}_{1}^{\beta}(E)}+\nu_{\alpha, \beta}(E)=\frac{d}{H \cdot \operatorname{ch}_{1}^{\beta}(E)}+\nu_{\alpha, \beta}\left(\mathcal{I}_{C}\right) \\
& >\frac{d}{H \cdot \operatorname{ch}_{1}^{\beta}\left(\mathcal{O}_{X}\right)}+\nu_{\alpha, \beta}\left(\mathcal{I}_{C}\right)=\nu_{\alpha, \beta}\left(\mathcal{O}_{X}\right)
\end{aligned}
$$

because $\mathcal{O}_{X}$ is stable in the whole $(\alpha, \beta)$-plane.

Lemma 3.13. The equation of the semidisk $Q_{\alpha, \beta}\left(\mathcal{I}_{C}\right) \leqslant 0$ is given by

$$
\alpha^{2}+\left(\beta+\frac{3 e}{2 d}\right)^{2}=\frac{9 e^{2}-8 d^{3}}{4 d^{2}} .
$$




\section{E. MACRÌ AND B. SCHMIDT}

Assume that $r$ is a positive integer such that

$$
e^{2}>\frac{2(2 r+1)^{2}}{9 r(r+1)} d^{3}
$$

Then $\mathcal{I}_{C}$ is destabilized via an exact sequence $0 \rightarrow E \rightarrow \mathcal{I}_{C} \rightarrow G \rightarrow 0$ defining a semicircular wall in tilt stability, where $0<\operatorname{ch}_{0}(E) \leqslant r$.

Proof. The claim about the equation is a straightforward calculation. For the second part observe that the hypothesis on $e^{2}$ implies

$$
\frac{9 e^{2}-8 d^{3}}{4 d^{2}}>\frac{d}{2 r(r+1)}>0 .
$$

Therefore, $\mathcal{I}_{C}$ has to destabilize at some point before $Q_{\alpha, \beta}\left(\mathcal{I}_{C}\right)<0$. Moreover, we can use Lemma 2.4 to conclude that $E$ cannot have rank greater than or equal to $r+1$.

Lemma 3.14. Assume that $e>\tilde{E}(d, k)$ holds.

(i) The object $\mathcal{I}_{C}$ is destabilized along a semicircular wall $W$ induced by $0 \rightarrow E \rightarrow \mathcal{I}_{C} \rightarrow G \rightarrow$ 0 , where $E$ is a reflexive sheaf. Let $H \cdot \operatorname{ch}(E)=(r, x, y, z)$. Then either $r=1$ or $r=2$.

(ii) Let $\mu_{0} \geqslant k$ be the unique integer such that either $d=\mu_{0}^{2}-f^{\prime}$ or $d=\mu_{0}\left(\mu_{0}+1\right)-f^{\prime}$, where $d \equiv-f^{\prime}\left(\bmod \mu_{0}\right)$ for $0 \leqslant f^{\prime}<\mu_{0}$. If $r=1$, then $k \leqslant-x \leqslant \mu_{0}$. If $r=2$, there are two possibilities.

(a) If $d=\mu_{0}^{2}-f^{\prime}$, then $x=-2 \mu_{0}$ or $x=-2 \mu_{0}+1$.

(b) If $d=\mu_{0}\left(\mu_{0}+1\right)-f^{\prime}$, then $x=-2 \mu_{0}$ or $x=-2 \mu_{0}-1$.

Proof. (i) If such a wall exists, then the reflexivity of $E$ follows immediately from Lemma 3.12. By Lemma 3.13, it is enough to show $e>(5 / 3 \sqrt{3}) d^{3 / 2}$ to get both $r \leqslant 2$ and the existence of the wall. Because of Lemma 3.11(i) and Lemma 3.10, it is enough to show that the function

$$
\varphi_{k}(d)=\frac{d^{2}}{2 k}+\frac{d k}{2}-\frac{k^{2}}{8}+\frac{k}{8}-\frac{5}{3 \sqrt{3}} d^{3 / 2}
$$

is non-negative whenever $d>k(k-1)$. The function $\varphi_{k}$ has a local maximum at $d_{0}=\frac{1}{3} k^{2}$, a local minimum at $d_{1}=\frac{3}{4} k^{2}$, and no other local extrema. Since $d_{0}<k(k-1)$, it is enough to check the positivity at $d_{1}$. Indeed, we have

$$
\varphi_{k}\left(\frac{3}{4} k^{2}\right)=\frac{k(k-2)^{2}}{32} \geqslant 0 .
$$

(ii) If $r=1$, then $E$ is a line bundle, and $k \leqslant-x$ follows from $\operatorname{Hom}\left(\mathcal{O}(-k+1), \mathcal{I}_{C}\right)=0$. By Lemma 3.11, we have $\tilde{E}\left(d, \mu_{0}\right) \leqslant \tilde{E}(d, k)$ and henceforth, $e>\tilde{E}\left(d, \mu_{0}\right)$. For any point $(\alpha, \beta) \in W$ with $\alpha>0$, we have $H \cdot \operatorname{ch}_{1}^{\beta}\left(\mathcal{I}_{C}\right)>H \cdot \operatorname{ch}_{1}^{\beta}(E)>0$. This can be rewritten as

$$
(r-1) \beta>x>r \beta \text {. }
$$

Note that if $Q_{0, \beta}\left(\mathcal{I}_{C}\right)<0$, then there is an $\alpha>0$ such that $(\alpha, \beta) \in W$. Since $x$ is an integer, the remaining claim is equivalent to showing the following two statements.

(a) If $d=\mu_{0}^{2}-f^{\prime}$, then $Q_{0,-\mu_{0}-1 / 2}\left(\mathcal{I}_{C}\right)<0$ and $Q_{0,2-2 \mu_{0}}\left(\mathcal{I}_{C}\right)<0$.

(b) If $d=\mu_{0}\left(\mu_{0}+1\right)-f^{\prime}$, then $Q_{0,-\mu_{0}-1}\left(\mathcal{I}_{C}\right)<0$ and $Q_{0,1-2 \mu_{0}}\left(\mathcal{I}_{C}\right)<0$.

The semidisk $Q_{0,-\mu_{0}-1}\left(\mathcal{I}_{C}\right)<0$ becomes smaller when $e$ decreases. Therefore, it is enough to check these inequalities for $e=\tilde{E}\left(d, \mu_{0}\right)$. 


\section{Derived CATEgories AND THE Genus of SPACE CURVES}

Assume $d=\mu_{0}^{2}-f^{\prime}$. Then

$$
Q_{0,-\mu_{0}-1 / 2}\left(\mathcal{I}_{C}\right)=-3 f^{\prime 2} \mu_{0}+2 f^{\prime} \mu_{0}^{2}-\mu_{0}^{3}+\frac{5}{2} f^{\prime 2}+f^{\prime} \mu_{0}+\frac{1}{2} \mu_{0}^{2}-2 f^{\prime} .
$$

This is a parabola in $f^{\prime}$ with maximum at

$$
f^{\prime}=\frac{2 \mu_{0}^{2}+\mu_{0}-2}{6 \mu_{0}-5} .
$$

From this maximum, we get

$$
Q_{0,-\mu_{0}-1 / 2}\left(\mathcal{I}_{C}\right) \leqslant-\frac{2\left(2 \mu_{0}+1\right)\left(\mu_{0}-1\right)^{3}}{6 \mu_{0}-5}<0 .
$$

Next, we have

$$
Q_{0,2-2 \mu_{0}}\left(\mathcal{I}_{C}\right)=-6 f^{\prime 2} \mu_{0}+8 f^{\prime} \mu_{0}^{2}-4 \mu_{0}^{3}+10 f^{\prime 2}-14 f^{\prime} \mu_{0}+8 \mu_{0}^{2}-2 f^{\prime} .
$$

This is a parabola in $f^{\prime}$ with maximum at

$$
f^{\prime}=\frac{4 \mu_{0}^{2}-7 \mu_{0}-1}{6 \mu_{0}-10} .
$$

From this maximum, we get

$$
Q_{0,2-2 \mu_{0}}\left(\mathcal{I}_{C}\right) \leqslant-\frac{\left(8 \mu_{0}^{2}-16 \mu_{0}-1\right)\left(\mu_{0}-1\right)^{2}}{6 \mu_{0}-10}<0
$$

unless $\mu_{0}=2$. In that case, we have $Q_{0,2-2 \mu_{0}}\left(\mathcal{I}_{C}\right)=-2 f^{\prime 2}+2 f^{\prime}$. However, we used $e=\tilde{E}(d, m)$ but could have used $e=\tilde{E}(d, m)+\frac{1}{8}$ to obtain a strict inequality.

Assume $d=\mu_{0}\left(\mu_{0}+1\right)-f^{\prime}$. Then

$$
Q_{0,-\mu_{0}-1}\left(\mathcal{I}_{C}\right)=-3 f^{\prime 2} \mu_{0}+2 f^{\prime} \mu_{0}^{2}-\mu_{0}^{3}+f^{\prime 2}+3 f^{\prime} \mu_{0}-2 \mu_{0}^{2}+f^{\prime}-\mu_{0} .
$$

This is a parabola in $f^{\prime}$ with maximum at

$$
f^{\prime}=\frac{2 \mu_{0}^{2}+3 \mu_{0}+1}{6 \mu_{0}-2} .
$$

From this maximum, we get

$$
Q_{0,-\mu_{0}-1}\left(\mathcal{I}_{C}\right) \leqslant-\frac{\left(8 \mu_{0}^{2}-8 \mu_{0}-1\right)\left(\mu_{0}+1\right)^{2}}{12 \mu_{0}-4}<0 .
$$

Finally, we have

$$
Q_{0,1-2 \mu_{0}}\left(\mathcal{I}_{C}\right)=-6 f^{\prime 2} \mu_{0}+8 f^{\prime} \mu_{0}^{2}-4 \mu_{0}^{3}+7 f^{\prime 2}-6 f^{\prime} \mu_{0}+\mu_{0}^{2}-5 f^{\prime}+5 \mu_{0} .
$$

This is a parabola in $f^{\prime}$ with maximum at

$$
f^{\prime}=\frac{8 \mu_{0}^{2}-6 \mu_{0}-5}{12 \mu_{0}-14} .
$$

From this maximum, we get

$$
Q_{0,1-2 \mu_{0}}\left(\mathcal{I}_{C}\right) \leqslant-\frac{\left(4 \mu_{0}^{2}+2 \mu_{0}-5\right)\left(4 \mu_{0}-5\right)\left(2 \mu_{0}-1\right)}{24 \mu_{0}-28}<0 .
$$

Lemma 3.15. Assume $d=\mu_{0}^{2}-f^{\prime}, d \equiv-f^{\prime}\left(\bmod \mu_{0}\right), 0 \leqslant f^{\prime}<\mu_{0}$, and

$$
e>\tilde{E}\left(d, \mu_{0}\right)=\mu_{0}^{3}+\frac{1}{2} f^{\prime 2}-2 f^{\prime} \mu_{0}+\frac{1}{2} f^{\prime} .
$$

Furthermore, suppose that $\mathcal{I}_{C}$ is destabilized at a wall $W$ induced by an exact sequence $0 \rightarrow$ $E \rightarrow \mathcal{I}_{C} \rightarrow G \rightarrow 0$, where $E$ is reflexive and tilt stable along $W$. 


\section{E. MACRÌ AND B. SCHMIDT}

(i) If $H \cdot \operatorname{ch}(E)=\left(2,-2 \mu_{0}, y, z\right)$, then the following hold:

(a) The Chern character of $E\left(\mu_{0}\right)$ is $\left(2,0,-\mu_{0}^{2}+y,-\frac{2}{3} \mu_{0}^{3}+\mu_{0} y+z\right)$.

(b) We have

$$
\frac{2 \mu_{0}^{4}-4 f^{\prime} \mu_{0}^{2}+3 f^{\prime 2} \mu_{0}+3 f^{\prime} \mu_{0}-4 f^{\prime 2}}{2\left(\mu_{0}^{2}-f^{\prime}\right)}<y \leqslant \mu_{0}^{2} \quad \text { and } \quad 0 \leqslant f^{\prime}<\frac{2 \mu_{0}^{2}-3 \mu_{0}}{3 \mu_{0}-4} .
$$

Moreover, if $\mu_{0} \in\{2,3,4\}$, then $y=\mu_{0}^{2}$.

(c) The inequality

$$
\begin{aligned}
e \leqslant & \frac{1}{2} \mu_{0}^{4}+f^{\prime} \mu_{0}^{2}-\frac{2}{3} \mu_{0}^{3}-\mu_{0}^{2} y+\frac{1}{2} f^{\prime 2}-2 f^{\prime} \mu_{0} \\
& +\frac{1}{2} \mu_{0}^{2}-f^{\prime} y+2 \mu_{0} y+\frac{1}{2} y^{2}+\frac{1}{2} f^{\prime}-\frac{1}{2} y+z
\end{aligned}
$$

holds.

(d) If $y=\mu_{0}^{2}$, then

$$
z \leqslant-\frac{1}{3} \mu_{0}^{3} \quad \text { and } \quad e \leqslant \mu_{0}^{3}+\frac{1}{2} f^{\prime 2}-2 f^{\prime} \mu_{0}+\frac{1}{2} f^{\prime}=\tilde{E}\left(d, \mu_{0}\right) .
$$

In particular, the wall $W$ cannot exist.

(e) If $y=\mu_{0}^{2}-\frac{1}{2}$, then

$$
z \leqslant-\frac{1}{3} \mu_{0}^{3}+\frac{1}{2} \mu_{0}+\frac{1}{6} \quad \text { and } \quad e \leqslant \mu_{0}^{3}+\frac{1}{2} f^{\prime 2}-2 f^{\prime} \mu_{0}+f^{\prime}-\frac{1}{2} \mu_{0}+\frac{13}{24} .
$$

In particular, the wall $W$ cannot exist.

(f) If $y \leqslant \mu_{0}^{2}-1$, then

$$
\begin{gathered}
z \leqslant \frac{1}{2} \mu_{0}^{4}+\frac{2}{3} \mu_{0}^{3}-\mu_{0}^{2} y-\mu_{0} y+\frac{1}{2} y^{2}+\frac{5}{24} \\
e \leqslant \mu_{0}^{4}+f^{\prime} \mu_{0}^{2}-2 \mu_{0}^{2} y+\frac{1}{2} f^{\prime 2}-2 f^{\prime} \mu_{0}+\frac{1}{2} \mu_{0}^{2}-f^{\prime} y+\mu_{0} y+y^{2}+\frac{1}{2} f^{\prime}-\frac{1}{2} y+\frac{5}{24} .
\end{gathered}
$$

In particular, the wall $W$ cannot exist.

(ii) If $H \cdot \operatorname{ch}(E)=\left(2,-2 \mu_{0}+1, y, z\right)$, then the following hold:

(a) The Chern character of $E\left(\mu_{0}-1\right)$ is given by

$$
\left(2,-1,-\mu_{0}^{2}+\mu_{0}+y,-\frac{2}{3} \mu_{0}^{3}+\frac{3}{2} \mu_{0}^{2}+\mu_{0} y-\mu_{0}-y+z+\frac{1}{6}\right) .
$$

(b) The second Chern character of $E$ satisfies

$$
\frac{4 \mu_{0}^{4}-8 f^{\prime} \mu_{0}^{2}-6 \mu_{0}^{3}-11 f^{\prime 2}+6 f^{\prime 2} \mu_{0}+18 f^{\prime} \mu_{0}-3 f^{\prime}}{4\left(\mu_{0}^{2}-f^{\prime}\right)}<y \leqslant \mu_{0}^{2}+f^{\prime}-2 \mu_{0}+\frac{1}{2} .
$$

Moreover, in the case $\mu_{0}=2$ we have $f^{\prime} \geqslant 1$.

(c) The inequality

$$
\begin{aligned}
e \leqslant & \frac{1}{2} \mu_{0}^{4}+f^{\prime} \mu_{0}^{2}-\frac{8}{3} \mu_{0}^{3}-\mu_{0}^{2} y+\frac{1}{2} f^{\prime 2}-4 f^{\prime} \mu_{0}+6 \mu_{0}^{2} \\
& -f^{\prime} y+4 \mu_{0} y+\frac{1}{2} y^{2}+2 f^{\prime}-4 \mu_{0}-2 y+z+\frac{17}{24}
\end{aligned}
$$

holds.

(d) We have

$$
\begin{gathered}
z \leqslant \frac{1}{2} \mu_{0}^{4}-\frac{1}{3} \mu_{0}^{3}-\mu_{0}^{2} y+\frac{1}{2} y^{2}+\frac{1}{24} \\
e \leqslant \mu_{0}^{4}+f^{\prime} \mu_{0}^{2}-3 \mu_{0}^{3}-2 \mu_{0}^{2} y+\frac{1}{2} f^{\prime 2}-4 f^{\prime} \mu_{0}+6 \mu_{0}^{2} \\
-f^{\prime} y+4 \mu_{0} y+y^{2}+2 f^{\prime}-4 \mu_{0}-2 y+\frac{3}{4} .
\end{gathered}
$$

In particular, the wall $W$ cannot exist.

Proof. (i) Assume $H \cdot \operatorname{ch}(E)=\left(2,-2 \mu_{0}, y, z\right)$. 


\section{Derived CATEGories AND THE GENus of SPACE CURVES}

(a) This part is a straightforward calculation.

(b) By Theorem 3.6, we get $-\mu_{0}^{2}+y \leqslant 0$; that is, $y \leqslant \mu_{0}^{2}$. The center of $W\left(E, \mathcal{I}_{C}\right)$ is given by

$$
s\left(E, \mathcal{I}_{C}\right)=\frac{-2 \mu_{0}^{2}+2 f^{\prime}-y}{2 \mu_{0}} .
$$

The semidisk $Q_{\alpha, \beta}\left(\mathcal{I}_{C}\right)<0$ becomes smaller when $e$ decreases, and we can bound it from below by setting $e=\tilde{E}\left(d, \mu_{0}\right)$. Therefore, the center $s_{Q}$ of this semidisk satisfies

$$
s_{Q}<\frac{-6 \mu_{0}^{3}-3 f^{\prime 2}+12 f^{\prime} \mu_{0}-3 f}{4 \mu_{0}^{2}-4 f^{\prime}} .
$$

The lower bound on $y$ is a consequence of the fact that $W$ is outside of $Q_{\alpha, \beta}\left(\mathcal{I}_{C}\right)<0$; that is, $s\left(E, \mathcal{I}_{C}\right) \leqslant s_{Q}$. Comparing the lower and upper bound for $y$ leads to the bound on $f^{\prime}$. If $\mu_{0} \in\{2,3,4\}$, then

$$
\frac{2 \mu_{0}^{4}-4 f^{\prime} \mu_{0}^{2}+3 f^{\prime 2} \mu_{0}+3 f^{\prime} \mu_{0}-4 f^{\prime 2}}{2\left(\mu_{0}^{2}-f^{\prime}\right)}>\mu_{0}^{2}-\frac{1}{2} .
$$

(c) This inequality on $e$ is an application of Proposition 3.2 to the quotient $G$.

(d) Assume $y=\mu_{0}^{2}$. The upper bound on $z$ follows from Theorem 3.6 applied to $E\left(\mu_{0}\right)$. The bound on $e$ is a direct consequence of applying the bound on $z$ to the previous upper bound for $e$.

(e) Assume $y=\mu_{0}^{2}-\frac{1}{2}$. The upper bound on $z$ follows from Theorem 3.6 applied to $E\left(\mu_{0}\right)$. The bound on $e$ is a direct consequence of applying the bound on $z$ to the previous upper bound for $e$. Indeed, for $\mu_{0} \neq 2$, we can use

$$
f^{\prime}<\frac{2 \mu_{0}^{2}-3 \mu_{0}}{3 \mu_{0}-4}
$$

to get

$$
\mu_{0}^{3}+\frac{1}{2} f^{\prime 2}-2 f^{\prime} \mu_{0}+f^{\prime}-\frac{1}{2} \mu_{0}+\frac{13}{24} \leqslant \mu_{0}^{3}+\frac{1}{2} f^{\prime 2}-2 f^{\prime} \mu_{0}+\frac{1}{2} f^{\prime} .
$$

(f) Assume $y \leqslant \mu_{0}^{2}-1$. The upper bound on $z$ follows from Theorem 3.6 applied to $E\left(\mu_{0}\right)$. The bound on $e$ is a direct consequence of applying the bound on $z$ to the previous upper bound for $e$. We are left to show that this inequality implies $e \leqslant \tilde{E}\left(d, \mu_{0}\right)$. This can be done by showing that the function

$$
\begin{aligned}
& \tilde{E}\left(d, \mu_{0}\right)-\left(\mu_{0}^{4}+f^{\prime} \mu_{0}^{2}-2 \mu_{0}^{2} y+\frac{1}{2} f^{\prime 2}-2 f^{\prime} \mu_{0}+\frac{1}{2} \mu_{0}^{2}-f^{\prime} y+\mu_{0} y+y^{2}+\frac{1}{2} f^{\prime}-\frac{1}{2} y+\frac{5}{24}\right) \\
& \quad=-\mu_{0}^{4}-f^{\prime} \mu_{0}^{2}+\mu_{0}^{3}+2 \mu_{0}^{2} y-\frac{1}{2} \mu_{0}^{2}+f^{\prime} y-\mu_{0} y-y^{2}+\frac{1}{2} y-\frac{5}{24}=: \varphi\left(y, f^{\prime}, \mu_{0}\right)
\end{aligned}
$$

is non-negative. This function is a parabola in $y$ with maximum. The maximum occurs at

$$
y_{0}=\mu_{0}^{2}+\frac{1}{2} f^{\prime}-\frac{1}{2} \mu_{0}+\frac{1}{4} .
$$

We will show that $y_{0}$ lies before our range for $y$ and therefore, $\varphi_{f^{\prime}, \mu_{0}}$ has a minimum for $y=\mu^{2}-1$. Indeed, we can compute

$$
\begin{aligned}
\psi\left(\mu_{0}, f^{\prime}\right) & :=\frac{2 \mu_{0}^{4}-4 f^{\prime} \mu_{0}^{2}+3 f^{\prime 2} \mu_{0}+3 f^{\prime} \mu_{0}-4 f^{\prime 2}}{2\left(\mu_{0}^{2}-f^{\prime}\right)}-y_{0} \\
& =\frac{6 f^{\prime 2} \mu_{0}-6 f^{\prime} \mu_{0}^{2}+2 \mu_{0}^{3}-6 f^{\prime 2}+4 f^{\prime} \mu_{0}-\mu_{0}^{2}+f^{\prime}}{4\left(\mu_{0}^{2}-f^{\prime}\right)} .
\end{aligned}
$$

The numerator is a parabola in $f^{\prime}$ with minimum at

$$
f_{0}^{\prime}=\frac{6 \mu_{0}^{2}-4 \mu_{0}-1}{12\left(\mu_{0}-1\right)}
$$




\section{E. MACRÌ AND B. SCHMIDT}

and

$$
\psi\left(\mu_{0}, f_{0}^{\prime}\right)=\frac{12 \mu_{0}^{4}-24 \mu_{0}^{3}+20 \mu_{0}^{2}-8 \mu_{0}-1}{8\left(6 \mu_{0}^{2}-6 \mu_{0}-1\right)\left(2 \mu_{0}-1\right)}>0 .
$$

Finally, we get

$$
\varphi\left(y, f^{\prime}, \mu_{0}\right) \geqslant \varphi\left(\mu_{0}^{2}-1, f^{\prime}, \mu_{0}\right)=-f^{\prime}+\mu_{0}-\frac{41}{24} \geqslant 0,
$$

due to the upper bound on $f^{\prime}$ and $\mu_{0} \geqslant 5$.

(ii) Assume $H \cdot \operatorname{ch}(E)=\left(2,-2 \mu_{0}+1, y, z\right)$.

(a) This part is a straightforward calculation.

(b) The inequality $\Delta(G) \geqslant 0$ is equivalent to $y \leqslant \mu_{0}^{2}+f^{\prime}-2 \mu_{0}+\frac{1}{2}$. The center of $W\left(E, \mathcal{I}_{C}\right)$ is given by

$$
s\left(E, \mathcal{I}_{C}\right)=\frac{-2 \mu_{0}^{2}+2 f^{\prime}-y}{2 \mu_{0}-1} .
$$

The semidisk $Q_{\alpha, \beta}\left(\mathcal{I}_{C}\right)<0$ becomes smaller when $e$ decreases, and we can bound it from below by setting $e=\tilde{E}\left(d, \mu_{0}\right)$. Therefore, the center $s_{Q}$ of this semidisk satisfies

$$
s_{Q}<\frac{-6 \mu_{0}^{3}-3 f^{\prime 2}+12 f^{\prime} \mu_{0}-3 f}{4 \mu_{0}^{2}-4 f^{\prime}} .
$$

The lower bound on $y$ is then obtained from the fact that $W$ is outside this lower bound of the semidisk; that is, $s\left(E, \mathcal{I}_{C}\right)<s_{Q}$. Finally, if $\mu_{0}=2$, then comparing the upper and lower bound on $y$ leads to $f^{\prime} \geqslant 1$.

(c) This inequality on $e$ is a direct application of Proposition 3.2 to the quotient $G$.

(d) The upper bound on $z$ follows from Theorem 3.6 applied to $E\left(\mu_{0}-1\right)$. The bound on $e$ is a direct consequence of estimating $z$ in the previous upper bound for $e$. We have to maximize the function

$$
\begin{aligned}
\varphi\left(y, f^{\prime}, \mu_{0}\right):= & \mu_{0}^{4}+f^{\prime} \mu_{0}^{2}-3 \mu_{0}^{3}-2 \mu_{0}^{2} y+\frac{1}{2} f^{2}-4 f^{\prime} \mu_{0}+6 \mu_{0}^{2} \\
& -f^{\prime} y+4 \mu_{0} y+y^{2}+2 f^{\prime}-4 \mu_{0}-2 y+\frac{3}{4} .
\end{aligned}
$$

This function is a parabola in $y$ with minimum at

$$
y_{0}=\mu_{0}^{2}+\frac{1}{2} f^{\prime}-2 \mu_{0}+1 .
$$

We will show that $y_{0}$ lies before our range for $y$, and, therefore, the maximum occurs at $y=$ $\mu_{0}^{2}+f^{\prime}-2 \mu_{0}+\frac{1}{2}$. Let

$$
\begin{aligned}
\psi\left(\mu_{0}, f^{\prime}\right) & :=\frac{4 \mu_{0}^{4}-8 f^{\prime} \mu_{0}^{2}-6 \mu_{0}^{3}-11 f^{\prime 2}+6 f^{\prime 2} \mu_{0}+18 f^{\prime} \mu_{0}-3 f^{\prime}}{4\left(\mu_{0}^{2}-f^{\prime}\right)}-y_{0} \\
& =\frac{6 f^{\prime 2} \mu_{0}-6 f^{\prime} \mu_{0}^{2}+2 \mu_{0}^{3}-9 f^{\prime 2}+10 f^{\prime} \mu_{0}-4 \mu_{0}^{2}+f^{\prime}}{4\left(\mu_{0}^{2}-f^{\prime}\right)} .
\end{aligned}
$$

The numerator is a parabola in $f^{\prime}$ with minimum at

$$
f_{0}^{\prime}=\frac{6 \mu_{0}^{2}-10 \mu_{0}-1}{12 \mu_{0}-18}
$$

and

$$
\psi\left(\mu_{0}, f_{0}^{\prime}\right)=\frac{12 \mu_{0}^{4}-48 \mu_{0}^{3}+56 \mu_{0}^{2}-20 \mu_{0}-1}{8\left(12 \mu_{0}^{3}-24 \mu_{0}^{2}+10 \mu_{0}+1\right)},
$$




\section{DERIVED CATEGORIES AND THE GENUS OF SPACE CURVES}

which is positive for $\mu_{0} \geqslant 3$. When $\mu_{0}=2$, then we get $f_{0}^{\prime}=\frac{1}{2}$ but $f^{\prime} \geqslant 1$. Then, $\psi(2,1)=0$. Finally, we get

$$
\varphi\left(y, f^{\prime}, \mu_{0}\right) \geqslant \varphi\left(\mu_{0}^{2}+f^{\prime}-2 \mu_{0}+\frac{1}{2}, f^{\prime}, \mu_{0}\right)=\tilde{E}\left(d, \mu_{0}\right) .
$$

Lemma 3.16. Assume $d=\mu_{0}\left(\mu_{0}+1\right)-f^{\prime}, d \equiv-f^{\prime}\left(\bmod \mu_{0}\right), 0 \leqslant f^{\prime}<\mu_{0}$, and

$$
e>\tilde{E}\left(d, \mu_{0}\right)=\mu_{0}^{3}+\frac{1}{2} f^{\prime 2}-2 f^{\prime} \mu_{0}+\frac{3}{2} \mu_{0}^{2}-\frac{1}{2} f^{\prime}+\frac{1}{2} \mu_{0} .
$$

Furthermore, suppose that $\mathcal{I}_{C}$ is destabilized at a wall $W$ induced by an exact sequence $0 \rightarrow$ $E \rightarrow \mathcal{I}_{C} \rightarrow G \rightarrow 0$, where $E$ is reflexive and tilt stable along $W$.

(i) If $H \cdot \operatorname{ch}(E)=\left(2,-2 \mu_{0}, y, z\right)$, then the following hold:

(a) The Chern character of $E\left(\mu_{0}\right)$ is given by $\left(2,0,-\mu_{0}^{2}+y,-\frac{2}{3} \mu_{0}^{3}+\mu_{0} y+z\right)$.

(b) We have

$$
\frac{2 \mu_{0}^{4}+3 f^{\prime 2} \mu_{0}-4 f^{\prime} \mu_{0}^{2}+\mu_{0}^{3}-4 f^{\prime 2}+5 f \mu_{0}-\mu_{0}^{2}}{2\left(\mu_{0}^{2}-f^{\prime}+\mu_{0}\right)}<y \leqslant \mu_{0}^{2}+f^{\prime}-\mu_{0}<\mu_{0}^{2} .
$$

If $y=\mu_{0}^{2}-\frac{1}{2}$, then $f^{\prime}=\mu_{0}-\frac{1}{2}$.

(c) The following inequality holds:

$$
\begin{aligned}
e \leqslant & \frac{1}{2} \mu_{0}^{4}+f^{\prime} \mu_{0}^{2}-\frac{5}{3} \mu_{0}^{3}-\mu_{0}^{2} y+\frac{1}{2} f^{\prime 2}-3 f^{\prime} \mu_{0}+3 \mu_{0}^{2} \\
& -f^{\prime} y+3 \mu_{0} y+\frac{1}{2} y^{2}+\frac{1}{2} f^{\prime}-\frac{1}{2} \mu_{0}-\frac{1}{2} y+z .
\end{aligned}
$$

(d) If $y=\mu_{0}^{2}-\frac{1}{2}$, then $f^{\prime}=\mu_{0}-\frac{1}{2}$ and

$$
z \leqslant-\frac{1}{3} \mu_{0}^{3}+\frac{1}{2} \mu_{0}^{2}+\frac{1}{6} \quad \text { and } \quad e \leqslant \mu_{0}^{3}+\frac{1}{2} \mu_{0}^{2}+\frac{1}{6} .
$$

In particular, the wall $W$ cannot exist.

(e) If $y \leqslant \mu_{0}^{2}-1$, then

$$
\begin{aligned}
z \leqslant & \frac{1}{2} \mu_{0}^{4}+\frac{2}{3} \mu_{0}^{3}-\mu_{0}^{2} y-\mu_{0} y+\frac{1}{2} y^{2}+\frac{5}{24} \\
e \leqslant & \mu_{0}^{4}+f^{\prime} \mu_{0}^{2}-\mu_{0}^{3}-2 \mu_{0}^{2} y+\frac{1}{2} f^{\prime 2}-3 f^{\prime} \mu_{0}+3 \mu_{0}^{2}-f^{\prime} y \\
& +2 \mu_{0} y+y^{2}+\frac{1}{2} f^{\prime}-\frac{1}{2} \mu_{0}-\frac{1}{2} y+\frac{5}{24} .
\end{aligned}
$$

In particular, the wall $W$ cannot exist.

(ii) If $H \cdot \operatorname{ch}(E)=\left(2,-2 \mu_{0}-1, y, z\right)$, then the following hold:

(a) The Chern character of $E\left(\mu_{0}\right)$ is given by $\left(2,-1,-\mu_{0}^{2}-\mu_{0}+y,-\frac{2}{3} \mu_{0}^{3}-\frac{1}{2} \mu_{0}^{2}+\mu_{0} y+z\right)$.

(b) We have

$$
\frac{4 \mu_{0}^{4}+6 f^{\prime 2} \mu_{0}-8 f^{\prime} \mu_{0}^{2}+8 \mu_{0}^{3}-5 f^{\prime 2}-2 f^{\prime} \mu_{0}+7 \mu_{0}^{2}-3 f^{\prime}+3 \mu_{0}}{4\left(\mu_{0}^{2}-f^{\prime}+\mu_{0}\right)}<y \leqslant \mu_{0}^{2}+\mu_{0} .
$$

(c) We have

$$
e \leqslant \frac{1}{2} \mu_{0}^{4}+f^{\prime} \mu_{0}^{2}+\frac{1}{3} \mu_{0}^{3}-\mu_{0}^{2} y+\frac{1}{2} f^{\prime 2}-f^{\prime} \mu_{0}+\frac{1}{2} \mu_{0}^{2}-f^{\prime} y+\mu_{0} y+\frac{1}{2} y^{2}+z+\frac{1}{24} .
$$

(d) We have

$$
\begin{aligned}
& z \leqslant \frac{1}{2} \mu_{0}^{4}+\frac{5}{3} \mu_{0}^{3}-\mu_{0}^{2} y+2 \mu_{0}^{2}-2 \mu_{0} y+\frac{1}{2} y^{2}+\mu_{0}-y+\frac{5}{24} \\
& e \leqslant \mu_{0}^{4}+f^{\prime} \mu_{0}^{2}+2 \mu_{0}^{3}-2 \mu_{0}^{2} y+\frac{1}{2} f^{\prime 2}-f^{\prime} \mu_{0}+\frac{5}{2} \mu_{0}^{2}-f^{\prime} y-\mu_{0} y+y^{2}+\mu_{0}-y+\frac{1}{4} .
\end{aligned}
$$

In particular, the wall $W$ cannot exist.

Proof. (i) Assume $H \cdot \operatorname{ch}(E)=\left(2,-2 \mu_{0}, y, z\right)$. 


\section{E. MACRÌ AND B. SCHMIDT}

(a) This part is a straightforward calculation.

(b) The inequality $\Delta(G) \geqslant 0$ is equivalent to $y \leqslant \mu_{0}^{2}+f^{\prime}-\mu_{0}$. The semicircular wall $W\left(E, \mathcal{I}_{C}\right)$ has center

$$
s\left(E, \mathcal{I}_{C}\right)=-\frac{2 \mu_{0}^{2}-2 f^{\prime}+2 \mu_{0}+y}{2 \mu_{0}}
$$

and the open semidisk $Q_{\alpha, \beta}\left(\mathcal{I}_{C}\right)<0$ has center

$$
s_{Q}=-\frac{3 e}{2\left(\mu_{0}^{2}+\mu_{0}-f^{\prime}\right)} .
$$

Since this semidisks center increases when $e$ decreases, the semidisk itself becomes smaller in that case, and we can bound it from below by setting $e=\tilde{E}\left(d, \mu_{0}\right)$. The lower bound on $y$ is then obtained from the fact that $W$ is outside this semidisk; that is, $s\left(E, \mathcal{I}_{C}\right) \leqslant s_{Q}$. If $y=\mu_{0}^{2}-\frac{1}{2}$, then $y \leqslant \mu_{0}^{2}+f^{\prime}-\mu_{0}$ implies $f^{\prime}=\mu_{0}-\frac{1}{2}$.

(c) This inequality on $e$ is a direct application of Proposition 3.2 to the quotient $G$.

(d) Assume $y=\mu_{0}^{2}-\frac{1}{2}$ and $f^{\prime}=\mu_{0}-\frac{1}{2}$. The upper bound on $z$ follows from Theorem 3.6 applied to $E\left(\mu_{0}\right)$. The bound on $e$ is a direct consequence of estimating $z$ in the previous upper bound for $e$. Indeed,

$$
e \leqslant \mu_{0}^{3}+\frac{1}{2} \mu_{0}^{2}+\frac{1}{6}<\mu_{0}^{3}+\frac{1}{2} \mu_{0}^{2}+\frac{3}{8}=\tilde{E}\left(d, \mu_{0}\right) .
$$

(e) Assume $y \leqslant \mu_{0}^{2}-1$. The upper bound on $z$ follows from Theorem 3.6 applied to $E\left(\mu_{0}\right)$. The bound on $e$ is a direct consequence of estimating $z$ in the previous upper bound for $e$. We are left to show that this inequality implies $e \leqslant \tilde{E}\left(d, \mu_{0}\right)$. This can be done by showing that the function

$$
\begin{aligned}
\varphi\left(y, f^{\prime}, \mu_{0}\right)= & \tilde{E}\left(d, \mu_{0}\right)-\left(\mu_{0}^{4}+f^{\prime} \mu_{0}^{2}-\mu_{0}^{3}-2 \mu_{0}^{2} y+\frac{1}{2} f^{\prime 2}-3 f^{\prime} \mu_{0}\right. \\
& \left.+3 \mu_{0}^{2}-f^{\prime} y+2 \mu_{0} y+y^{2}+\frac{1}{2} f^{\prime}-\frac{1}{2} \mu_{0}-\frac{1}{2} y+\frac{5}{24}\right) \\
= & -\mu_{0}^{4}-f^{\prime} \mu_{0}^{2}+2 \mu_{0}^{3}+2 \mu_{0}^{2} y+f^{\prime} \mu_{0}-\frac{3}{2} \mu_{0}^{2}+f^{\prime} y \\
& \quad-2 \mu_{0} y-y^{2}-f^{\prime}+\mu_{0}+\frac{1}{2} y-\frac{5}{24} .
\end{aligned}
$$

is positive. This function is a parabola in $y$ with maximum. The maximum occurs at $y_{0}=$ $\mu_{0}^{2}+\frac{1}{2} f^{\prime}-\mu_{0}+\frac{1}{4}$. We will show that $y_{0}$ lies before our range for $y$ and that, therefore, the minimum in our range occurs for $y=\mu^{2}+f^{\prime}-\mu_{0}$. We can compute

$$
\begin{aligned}
\psi\left(\mu_{0}, f^{\prime}\right) & :=\frac{2 \mu_{0}^{4}+3 f^{\prime 2} \mu_{0}-4 f^{\prime} \mu_{0}^{2}+\mu_{0}^{3}-4 f^{\prime 2}+5 f \mu_{0}-\mu_{0}^{2}}{2\left(\mu_{0}^{2}-f^{\prime}+\mu_{0}\right)}-y_{0} \\
& =\frac{6 f^{\prime 2} \mu_{0}-6 f^{\prime} \mu_{0}^{2}+2 \mu_{0}^{3}-6 f^{\prime 2}+4 f^{\prime} \mu_{0}+\mu_{0}^{2}+f^{\prime}-\mu_{0}}{4\left(\mu_{0}^{2}-f^{\prime}+\mu_{0}\right)} .
\end{aligned}
$$

The numerator is a parabola in $f^{\prime}$ with minimum at

$$
f_{0}^{\prime}=\frac{6 \mu_{0}^{2}-4 \mu_{0}-1}{12 \mu_{0}-12}
$$

and

$$
\psi\left(\mu_{0}, f_{0}^{\prime}\right)=\frac{12 \mu_{0}^{4}+24 \mu_{0}^{3}-52 \mu_{0}^{2}+16 \mu_{0}-1}{96 \mu_{0}^{3}-48 \mu_{0}^{2}-64 \mu_{0}+1}>0 .
$$

Thus, $\varphi\left(y, f^{\prime}, \mu_{0}\right)$ has a minimum $y=\mu^{2}+f^{\prime}-\mu_{0}$, where

$$
\varphi\left(\mu^{2}+f^{\prime}-\mu_{0}, f^{\prime}, \mu_{0}\right)=\frac{1}{2} \mu_{0}-\frac{1}{2} f^{\prime}-\frac{5}{24}>0 .
$$

(ii) Assume $H \cdot \operatorname{ch}(E)=\left(2,-2 \mu_{0}-1, y, z\right)$. 


\section{Derived CATEGories AND THE GENus of SPACE CURVES}

(a) This part is a straightforward calculation.

(b) By Theorem 3.6 applied to $E\left(\mu_{0}\right)$, we have $y \leqslant \mu_{0}^{2}+\mu_{0}$. The semicircular wall $W$ has center

$$
s\left(E, \mathcal{I}_{C}\right)=-\frac{2 \mu_{0}^{2}-2 f^{\prime}+2 \mu_{0}+y}{2 \mu_{0}+1},
$$

and the open semidisk $Q_{\alpha, \beta}\left(\mathcal{I}_{C}\right)<0$ has center

$$
s_{Q}=-\frac{3 e}{2\left(\mu_{0}^{2}+\mu_{0}-f^{\prime}\right)} .
$$

Since this semidisk's center increases when $e$ decreases, the semidisk itself becomes smaller in that case, and we can bound it from below by setting $e=\tilde{E}\left(d, \mu_{0}\right)$. The lower bound on $y$ is a consequence of the fact that $W$ is outside this semidisk; that is, $s\left(E, \mathcal{I}_{C}\right) \leqslant s_{Q}$.

(c) This inequality on $e$ is a direct application of Proposition 3.2 to the quotient $G$.

(d) The upper bound on $z$ follows from Theorem 3.6 applied to $E\left(\mu_{0}\right)$. The bound on $e$ is a direct consequence of estimating $z$ in the previous upper bound for $e$. We have to maximize

$$
\varphi\left(y, f^{\prime}, \mu_{0}\right)=\mu_{0}^{4}+f^{\prime} \mu_{0}^{2}+2 \mu_{0}^{3}-2 \mu_{0}^{2} y+\frac{1}{2} f^{\prime 2}-f^{\prime} \mu_{0}+\frac{5}{2} \mu_{0}^{2}-f^{\prime} y-\mu_{0} y+y^{2}+\mu_{0}-y+\frac{1}{4} .
$$

This function is a parabola in $y$ with minimum at $y_{0}=\mu_{0}^{2}+\frac{1}{2} f^{\prime}+\frac{1}{2} \mu_{0}+\frac{1}{2}$. We will show that $y_{0}$ lies before our range for $y$ and that, therefore, the maximum occurs at $y=\mu_{0}^{2}+\mu_{0}$. We want to show that

$$
\begin{aligned}
\psi\left(\mu_{0}, f^{\prime}\right) & :=\frac{4 \mu_{0}^{4}+6 f^{\prime 2} \mu_{0}-8 f^{\prime} \mu_{0}^{2}+8 \mu_{0}^{3}-5 f^{\prime 2}-2 f^{\prime} \mu_{0}+7 \mu_{0}^{2}-3 f^{\prime}+3 \mu_{0}}{4\left(\mu_{0}^{2}-f^{\prime}+\mu_{0}\right)}-y_{0} \\
& =\frac{6 f^{\prime 2} \mu_{0}-6 f^{\prime} \mu_{0}^{2}+2 \mu_{0}^{3}-3 f^{\prime 2}-2 f^{\prime} \mu_{0}+3 \mu_{0}^{2}-f^{\prime}+\mu_{0}}{4\left(\mu_{0}^{2}-f^{\prime}+\mu_{0}\right)}
\end{aligned}
$$

is non-negative. The numerator is a parabola in $f^{\prime}$ with minimum at

$$
f_{0}^{\prime}=\frac{6 \mu_{0}^{2}+2 \mu_{0}+1}{12 \mu_{0}-6}
$$

and

Finally,

$$
\psi\left(\mu_{0}, f_{0}^{\prime}\right)=\frac{12 \mu_{0}^{4}+24 \mu_{0}^{3}-28 \mu_{0}^{2}-16 \mu_{0}-1}{8\left(12 \mu_{0}^{3}-8 \mu_{0}-1\right)}>0
$$

$$
\varphi\left(\mu_{0}^{2}+\mu_{0}, f^{\prime}, \mu_{0}\right)=\mu_{0}^{3}+\frac{1}{2} f^{\prime 2}-2 f^{\prime} \mu_{0}+\frac{3}{2} \mu_{0}^{2}+\frac{1}{4} \leqslant \tilde{E}\left(d, \mu_{0}\right) .
$$

Proof of Theorem 3.1. By Lemma 3.14(i), we know that $\mathcal{I}_{C}$ is destabilized by an exact sequence $0 \rightarrow E \rightarrow \mathcal{I}_{C} \rightarrow G \rightarrow 0$, where $H \cdot \operatorname{ch}(E)=(r, x, y, z), E$ is reflexive, and $r=1$ or $r=2$.

Assume $r=1$. Then $E$ is a line bundle with $H \cdot \operatorname{ch}_{1}(E)=x$. and by Lemma 3.14(ii) we get $x \leqslant-k$ and $d>-x(-x-1)$. Then $H \cdot \operatorname{ch}(G)=\left(0,-x,-d-\frac{1}{2} x^{2}, e-\frac{1}{6} x^{3}\right)$, and a direct application of Theorem 3.4 gives $e \leqslant \tilde{E}(d,-x)$. By Lemma 3.11, we get $e \leqslant \tilde{E}(d, k)$, which is in contradiction to the assumption $e>\tilde{E}(d, k)$.

Assume $r=2$. As previously, let $\mu_{0} \geqslant k$ be the unique integer such that either $d=\mu_{0}^{2}-f^{\prime}$ or $d=\mu_{0}\left(\mu_{0}+1\right)-f^{\prime}$, where $d \equiv-f^{\prime}\left(\bmod \mu_{0}\right)$ and $0 \leqslant f^{\prime}<\mu_{0}$. Then Lemma 3.14(ii) implies that we are in any of the four situations described in Lemmas 3.15 and 3.16. These lemmas imply that $e \leqslant \tilde{E}\left(d, \mu_{0}\right)$. Since $\mu_{0} \geqslant k$, we again get a contradiction by using Lemma 3.11 to obtain $e \leqslant \tilde{E}(d, k)$. 


\section{E. MACRÌ AND B. SCHMIDT}

\section{Toward the Hartshorne-Hirschowitz conjecture}

In the previous section, we gave a new proof for the maximal genus of a degree $d$ curve $C \subset \mathbb{P}^{3}$ with $H^{0}\left(\mathcal{I}_{C}(k-1)\right)=0$ for some positive integer $k$ satisfying $d>k(k-1)$. The main open question is what happens for

$$
\frac{1}{3}\left(k^{2}+4 k+6\right) \leqslant d \leqslant k(k-1) .
$$

Let us rewrite the conjectural answer for this case in terms of the Chern character. Recall from the introduction that for any integer $c \in \mathbb{Z}$, we defined

$$
\delta(c)= \begin{cases}3 & \text { if } c=1,3 \\ 1 & \text { if } c \equiv 2(\bmod 3), \\ 0 & \text { otherwise. }\end{cases}
$$

Then, for any integers $k \geqslant 5$ and $f \in[k-1,2 k-5]$, we defined integers

$$
\begin{aligned}
& A(k, f)=\frac{1}{3}\left(k^{2}-k f+f^{2}-2 k+7 f+12+\delta(2 k-f-6)\right), \\
& B(k, f)=\frac{1}{3}\left(k^{2}-k f+f^{2}+6 f+11+\delta(2 k-f-7)\right) .
\end{aligned}
$$

Conjecture 4.1 (Hartshorne-Hirschowitz). Let $C \subset \mathbb{P}^{3}$ be an integral curve of degree $d$ such that $H^{0}\left(\mathcal{I}_{C}(k-1)\right)=0$ for some positive integer $k$. Assume $A(k, f) \leqslant d<A(k, f+1)$ for $f \in[k-1,2 k-6]$. Then

$$
\operatorname{ch}_{3}\left(\mathcal{I}_{C}\right) \leqslant E(d, k):=d(k+1)-\left(\begin{array}{c}
k+2 \\
3
\end{array}\right)+\left(\begin{array}{c}
f-k+4 \\
3
\end{array}\right)+h(d),
$$

where

$$
h(d)= \begin{cases}0 & \text { if } A(k, f) \leqslant d \leqslant B(k, f), \\ \frac{1}{2}(d-B(k, f))(d-B(k, f)+1) & \text { if } B(k, f) \leqslant d<A(k, f+1)) .\end{cases}
$$

The goal of this section is to prove the following result.

Theorem 4.2. Assume the hypothesis of Conjecture 4.1. Furthermore, let $A(k, f) \leqslant d \leqslant B(k, f)$, and assume that the base field has characteristic zero. If $\mathcal{I}_{C}$ is destabilized in tilt stability above or at the numerical wall $W\left(\mathcal{I}_{C}, \mathcal{O}(-f-4)[1]\right)$, then $\operatorname{ch}_{3}\left(\mathcal{I}_{C}\right) \leqslant E(d, k)$.

\subsection{Bounding sections of ideal sheaves}

The results in this section are elementary ingredients in the proof of Theorem 4.2.

Proposition 4.3. Let $Z \subset \mathbb{P}^{2}$ be a zero-dimensional subscheme of length $n$. Then

$$
h^{0}\left(\mathcal{I}_{Z}(l)\right) \leqslant\left(\begin{array}{c}
l+1 \\
2
\end{array}\right)
$$

for any integer $l<n$.

Proof. If $n=l+1$, then $\mathcal{O}_{\mathbb{P}^{2}}(l)$ is $l$-very ample (see [CG90] for details on this notion) and $h^{0}\left(\mathcal{I}_{Z}(l)\right)=\left(\begin{array}{c}l+1 \\ 2\end{array}\right)$. More generally, for $n>l+1$, the statement follows from a straightforward induction on $n$.

The following corollary will be crucial to the proof of the proposition. 


\section{Derived CATEgories AND THE Genus of SPACE CURVES}

Corollary 4.4. Let $C \subset \mathbb{P}^{3}$ be an arbitrary one-dimensional subscheme of degree $d$. Then

$$
h^{0}\left(\mathcal{I}_{C}(l)\right) \leqslant\left(\begin{array}{c}
l+2 \\
3
\end{array}\right)
$$

for any integer $l<d$.

Proof. The proof will be achieved by induction on $l$. Indeed, for $l \leqslant 0$ we have $h^{0}\left(\mathcal{I}_{C}(l)\right)=0$. Assume that we know that the statement holds for some $l>0$. Let $H \subset \mathbb{P}^{3}$ be a general plane such that the scheme-theoretic intersection $Z=H \cap C$ is zero-dimensional and of length $d$. We have a short exact sequence

$$
0 \rightarrow \mathcal{O}(l) \rightarrow \mathcal{O}(l+1) \rightarrow \mathcal{O}_{H}(l+1) \rightarrow 0 .
$$

By tensoring with $\mathcal{I}_{C}$, we get another short exact sequence

$$
0 \rightarrow \mathcal{I}_{C}(l) \rightarrow \mathcal{I}_{C}(l+1) \rightarrow \mathcal{I}_{C} \otimes \mathcal{O}_{H}(l+1)=\mathcal{I}_{Z / \mathbb{P}^{2}}(l+1) \rightarrow 0 .
$$

This sequence is exact on the left since the map $\mathcal{I}_{C}(l) \rightarrow \mathcal{I}_{C}(l+1)$ is injective by direct inspection. As a consequence, we can use Proposition 4.3 and the inductive hypothesis to obtain

$$
h^{0}\left(\mathcal{I}_{C}(l+1)\right) \leqslant h^{0}\left(\mathcal{I}_{C}(l)\right)+h^{0}\left(\mathcal{I}_{Z / \mathbb{P}^{2}}(l+1)\right) \leqslant\left(\begin{array}{c}
l+2 \\
3
\end{array}\right)+\left(\begin{array}{c}
l+2 \\
2
\end{array}\right)=\left(\begin{array}{c}
l+3 \\
3
\end{array}\right) .
$$

\subsection{Proof of Theorem 4.2}

LEMma 4.5. The objects $\mathcal{I}_{C}$ and $\mathcal{O}(-f-4)[1]$ are in the category $\operatorname{Coh}^{\beta}\left(\mathbb{P}^{3}\right)$ along the wall $W\left(\mathcal{I}_{C}, \mathcal{O}(-f-4)[1]\right)$. If $\mathcal{I}_{C}$ is destabilized by a map $\mathcal{I}_{C} \rightarrow \mathcal{O}(-f-4)[1]$, then Conjecture 4.1 holds for $C$.

Proof. The first claim holds if and only if $-f-4<\bar{\beta}\left(\mathcal{I}_{C}\right)=-\sqrt{2 d}$. Therefore, we have to show $\frac{1}{2}(f+4)^{2}-d>0$. By definition,

$$
\begin{aligned}
\frac{(f+4)^{2}}{2}-d & >\frac{(f+4)^{2}}{2}-A(k, f+1) \\
& \geqslant \frac{(f+4)^{2}}{2}-\frac{1}{3}\left(k^{2}-k(f+1)+(f+1)^{2}-2 k+7(f+1)+15\right) \\
& =\frac{1}{6} f^{2}+\frac{1}{3} f k-\frac{1}{3} k^{2}+f+k+\frac{1}{3} .
\end{aligned}
$$

The last term is a parabola in $f$ with minimum at $f=-k-3$. Since we have $f \geqslant k-1$, we can get a lower bound by setting $f=k-1$, where

$$
\frac{1}{6} f^{2}+\frac{1}{3} f k-\frac{1}{3} k^{2}+f+k+\frac{1}{3}=\frac{1}{6} k^{2}+\frac{4}{3} k-\frac{1}{2}>0 .
$$

Let $E$ be the kernel of the morphism $\mathcal{I}_{C} \rightarrow \mathcal{O}(-f-4)[1]$. The second claim immediately follows from Theorem 1.10 applied to $E(k-1)$.

Lemma 4.6. Assume $A(k, f) \leqslant d \leqslant B(k, f)$. Then walls for objects with Chern character $\operatorname{ch}\left(\mathcal{I}_{C}\right)$ above or at the numerical wall with $\mathcal{O}(-f-4)[1]$ are induced by a rank two subobject that is a reflexive sheaf.

Proof. Since $C$ is integral, we already showed in Lemma 3.12 that the destabilizing subobject has to be reflexive. All we have to show is that it is of rank two. The radius of $W\left(\mathcal{I}_{C}, \mathcal{O}(-f-4)[1]\right.$ is given by

$$
\rho^{2}=\rho\left(\mathcal{I}_{C}, \mathcal{O}(-f-4)[1]\right)^{2}=\left(\frac{f+4}{2}-\frac{d}{f+4}\right)^{2} .
$$




\section{E. MACRÌ AND B. SCHMIDT}

By Lemma 2.4, the inequality $\rho^{2}>\frac{1}{12} d$ will imply that the subobject has rank at most two. This inequality is equivalent to

$$
\left(\frac{3(f+4)}{4}-\frac{d}{f+4}\right)\left(\frac{f+4}{3}-\frac{d}{f+4}\right)>0 .
$$

This would follow from $d<\frac{1}{3}(f+4)^{2}$. The inequalities $k-1 \leqslant f \leqslant 2 k-6$ imply $\frac{1}{2} f+3 \leqslant k \leqslant f+1$. We know that

$$
d \leqslant A(k, f+1)-1 \leqslant \frac{f^{2}-f k+k^{2}+9 f-3 k+20}{3} .
$$

The right-hand side is a parabola in $k$ with minimum at $k=\frac{1}{2} f+\frac{3}{2}$. Therefore, we get an upper bound by setting $k=f+1$, which leads to

$$
d \leqslant \frac{f^{2}+7 f+18}{3}=\frac{(f+4)^{2}}{3}-\frac{f-2}{3}<\frac{(f+4)^{2}}{3} .
$$

Lastly, we have to rule out that $\mathcal{I}_{C}$ is destabilized by a line bundle. The largest point of the intersection of $W\left(\mathcal{I}_{C}, \mathcal{O}(-f-4)[1]\right.$ with the $\beta$-axis is given by $\beta_{0}=-2 d /(f+4)$. We are done if we can show that $\beta_{0}>-k$. This is equivalent to showing $d<\frac{1}{2}(f+4) k$. We can compute

$$
\begin{aligned}
\frac{(f+4) k}{2}-d & \geqslant \frac{(f+4) k}{2}-B(k, f) \\
& \geqslant \frac{(f+4) k}{2}-\frac{k^{2}-f k+f^{2}+6 f+14}{3} \\
& =-\frac{f^{2}}{3}+\frac{5 f k}{6}-\frac{k^{2}}{3}-2 f+2 k-\frac{14}{3} .
\end{aligned}
$$

The last term defines a parabola in $f$ with maximum. Its minimum has to be given at either $f=k-1$ or $f=2 k-6$. For $f=2 k-6$, we get

$$
\frac{(f+4) k}{2}-d \geqslant k-\frac{14}{3}>0,
$$

and for $f=k-1$, we get

$$
\frac{(f+4) k}{2}-d \geqslant \frac{k^{2}}{6}-\frac{k}{6}-3>0 .
$$

Lemma 4.7. Assume that $\mathcal{I}_{C}$ is destabilized at or above the numerical wall with $\mathcal{O}(-f-4)[1]$ but not by a quotient $\mathcal{I}_{C} \rightarrow \mathcal{O}(-f-4)[1]$. Furthermore, let $A(k, f) \leqslant d \leqslant B(k, f)$. Then $\mathcal{I}_{C}$ is destabilized by a quotient $\mathcal{I}_{C} \rightarrow G(-f-5)$ of rank -1 with $\operatorname{ch}_{1}(G)=0$. Moreover,

$$
\operatorname{ch}_{2}(G) \in\left[A(k, f+1)-d, \frac{f+5}{2}-\frac{d}{f+4}\right] \text {. }
$$

Proof. By Lemma 4.6, we know that $\mathcal{I}_{C}$ has to be destabilized by an exact sequence $0 \rightarrow E \rightarrow$ $\mathcal{I}_{C} \rightarrow G^{\prime} \rightarrow 0$ in $\operatorname{Coh}^{\beta}\left(\mathbb{P}^{3}\right)$, where $E$ has rank two and is a reflexive sheaf. Let $x=\operatorname{ch}_{1}\left(G^{\prime}\right)$, $G=G^{\prime}(x)$, and $y=\operatorname{ch}_{2}(G)$. Note that, by definition, $\operatorname{ch}_{1}(G)=0$. The wall $W\left(\mathcal{I}_{C}, \mathcal{O}(-f-4)[1]\right)$ intersects the $\beta$-axis at the two points

$$
\beta_{0}=-f-4 \quad \text { and } \quad \beta_{1}=-\frac{2 d}{f+4} .
$$

Since $W\left(\mathcal{I}_{C}, \mathcal{O}(-f-4)[1]\right)$ is less than or equal to $W\left(\mathcal{I}_{C}, G\right)$, we get

$$
0 \leqslant \operatorname{ch}_{1}^{\beta}(G(-x))=x+\beta \leqslant \operatorname{ch}_{1}^{\beta}\left(\mathcal{I}_{C}\right)=-\beta
$$




\section{Derived CATEGories AND THE GENus of SPACE CURVES}

for any $\beta$ such that there is a point $(\alpha, \beta) \in W\left(\mathcal{I}_{C}, \mathcal{O}(-f-4)[1]\right)$. In particular, we can choose both $\beta_{0}$ and $\beta_{1}$ in these inequalities to obtain $f+4 \leqslant x \leqslant 4 d /(f+4)$. The center of $W\left(\mathcal{I}_{C}, \mathcal{O}(-f-4)[1]\right)$ is given by

$$
s\left(\mathcal{I}_{C}, \mathcal{O}(-f-4)[1]\right)=-\frac{f+4}{2}-\frac{d}{f+4} .
$$

The center of $W\left(\mathcal{I}_{C}, G\right)$ is given by

$$
s\left(\mathcal{I}_{C}, G\right)=\frac{y-d}{x}-\frac{x}{2} .
$$

Therefore, the fact that $W\left(\mathcal{I}_{C}, \mathcal{O}(-f-4)[1]\right)$ is smaller than $W\left(\mathcal{I}_{C}, G\right)$ implies

$$
y \leqslant \frac{(2 d-x(f+4))(f+4-x)}{2(f+4)}=: \varphi_{d, f}(x) .
$$

We have

$$
\operatorname{ch}_{\leqslant 2}(E(k-1))=\left(2,2 k-x-2, k^{2}-k x+\frac{1}{2} x^{2}-d-2 k+x-y+1\right) .
$$

Since $H^{0}\left(\mathcal{I}_{C}(k-1)\right)=0$, we must have $H^{0}(E(k-1))=0$. Thus, we can apply Theorem 1.10 to get the following bound on $y$ :

$$
\begin{aligned}
y & \geqslant \frac{k^{2}}{3}-\frac{k x}{3}+\frac{x^{2}}{3}-d+\frac{2 k}{3}-\frac{x}{3}+\frac{\delta(2 k-x-2)}{3}=A(k, x-4)-d \\
& \geqslant \frac{k^{2}}{3}-\frac{k x}{3}+\frac{x^{2}}{3}-d+\frac{2 k}{3}-\frac{x}{3}=: \psi_{d, k}(x) .
\end{aligned}
$$

We will rule out specific values of $x$ by showing that $\varphi_{d, f}(x)<\psi_{d, k}(x)$. The first step in the proof is to show that if $\varphi_{d, f}(x)<\psi_{d, k}(x)$, then $\varphi_{d, f}\left(x^{\prime}\right)<\psi_{d, k}\left(x^{\prime}\right)$ for any $x^{\prime} \geqslant x$. This will be achieved by comparing the derivatives in $x$ that are given by

$$
\varphi_{d, f}^{\prime}(x)=x-\frac{d}{f+4}-\frac{f+4}{2} \quad \text { and } \quad \psi_{d, k}^{\prime}(x)=\frac{2 x}{3}-\frac{k}{3}-\frac{1}{3} .
$$

Combining this with $d \leqslant A(k, f+1)-1$ and $x \leqslant 4 d /(f+4)$, we get

$$
\begin{aligned}
\psi_{d, k}^{\prime}(x)-\varphi_{d, f}^{\prime}(x) & =\frac{f}{2}-\frac{k}{3}-\frac{x}{3}+\frac{5}{3}+\frac{d}{f+4} \\
& \geqslant \frac{f}{2}-\frac{k}{3}+\frac{5}{3}-\frac{d}{3(f+4)} \\
& \geqslant \frac{7 f^{2}-4 f k-2 k^{2}+48 f-18 k+80}{18(f+4)} .
\end{aligned}
$$

The numerator is a parabola in $f$ with minimum at $f=\frac{2}{7} k-\frac{24}{7}$. Therefore, it is enough to plug $f=k-1$ into the numerator, where indeed $k^{2}+20 k+39 \geqslant 0$. To summarize, we showed that it is enough to rule out $x=f+6$ by using $A(k, f) \leqslant d \leqslant B(k, f)$. In this case, the derivative of $\varphi$ by $d$ is larger than the derivative of $\psi$ by $d$. Therefore, we obtain $\varphi_{d, f}(f+6)<\psi_{d, k}(f+6)$ if this holds for any upper bound on $d$, for example,

$$
d \leqslant B(k, f) \leqslant \frac{k^{2}-k f+f^{2}+6 f+14}{3} .
$$

Using this upper bound for $d$, we get

$$
\psi_{d, k}(f+6)-\varphi_{d, f}(f+6) \geqslant \frac{2}{3} \cdot \frac{2 f^{2}-3 f k+k^{2}+9 f-8 k+10}{f+4} .
$$




\section{E. MACRÌ AND B. SCHMIDT}

The numerator is a parabola in $f$ with minimum at $f=\frac{3}{4} k-\frac{9}{4}$. Setting $f=k-1$ leads to

$$
2 f^{2}-3 f k+k^{2}+9 f-8 k+10 \geqslant 3>0 .
$$

Finally, the bounds on $y$ follow by setting $x=f+5$ in $\varphi$ and $A(k, x-4)-d$.

Lemma 4.8. Assume that $\mathcal{I}_{C}$ is destabilized at or above the numerical wall with $\mathcal{O}(-f-4)[1]$, by an exact sequence $0 \rightarrow E \rightarrow \mathcal{I}_{C} \rightarrow G(-f-5) \rightarrow 0$, where $\operatorname{ch}_{0}(G)=-1$ and $\operatorname{ch}_{1}(G)=0$. If $A(k, f) \leqslant d \leqslant B(k, f)$, then $h^{2}(E(k-1))=0$.

Proof. Let $y=\operatorname{ch}_{2}(G)$. We have

$$
\operatorname{ch}_{\leqslant 2}(E(k-1))=\left(2,2 k-f-7, \frac{1}{2} f^{2}-f k+k^{2}-d+6 f-7 k-y+\frac{37}{2}\right) .
$$

By Theorem 1.10, it is enough to show

$$
\begin{aligned}
\operatorname{ch}_{2}(E(k-1))-\left(\frac{1}{6} \operatorname{ch}_{1}(E(k-1))^{2}-\operatorname{ch}_{1}(E(k-1))-\frac{8}{3}-\frac{\delta(2 k-f-8)}{3}\right) \\
=\frac{1}{3} f^{2}-\frac{1}{3} f k+\frac{1}{3} k^{2}-d+\frac{8}{3} f-\frac{1}{3} k+\frac{\delta(2 k-f-8)}{3}-y+6
\end{aligned}
$$

is non-negative. By Lemma 4.7, we have

$$
y \leqslant \frac{f+5}{2}-\frac{d}{f+4} .
$$

Additionally, we can use $d \leqslant B(k, f)$ and $f \geqslant k-1$ to obtain

$$
\begin{aligned}
\frac{1}{3} f^{2} & -\frac{1}{3} f k+\frac{1}{3} k^{2}-d+\frac{8}{3} f-\frac{1}{3} k+\frac{\delta(2 k-f-8)}{3}-y+6 \\
& \geqslant \frac{3 f^{2}-4 f k+2 k^{2}+15 f-8 k+18+(2 f+8) \delta(2 k-f-8)-(2 f+6) \delta(2 k-f-7)}{6 f+24} \\
& \geqslant \frac{3 f^{2}-4 f k+2 k^{2}+9 f-8 k}{6 f+24} \geqslant 0 .
\end{aligned}
$$

Recall that for any $E \in \mathrm{D}^{\mathrm{b}}\left(\mathbb{P}^{3}\right)$, we defined the derived dual $\mathbb{D}(E)=\mathbf{R H o m}(E, \mathcal{O})[1]$.

Lemma 4.9. Let $E \in \operatorname{Coh}^{\beta}\left(\mathbb{P}^{3}\right)$ for some $\beta>0$ be a tilt semistable object for $\alpha \gg 0$ with $\operatorname{ch}(E)=(-1,0, d, e)$. Then there is a distinguished triangle

$$
\mathcal{I}_{C^{\prime}} \rightarrow \mathbb{D}(E) \rightarrow T[-1] \rightarrow \mathcal{I}_{C^{\prime}}[1],
$$

where $C^{\prime} \subset \mathbb{P}^{3}$ is a closed subscheme and $T$ is a sheaf supported in dimension zero. If $d>0$, then $\operatorname{dim} C^{\prime}=1$. If $d=0$, then $\operatorname{dim} C^{\prime}=0$.

Proof. Choose $\alpha \gg 0$ such that both $(\alpha, \beta)$ and $(\alpha,-\beta)$ are above the largest wall in tilt stability for walls with respect to $(-1,0, d)$ or $(1,0,-d)$. By Proposition 2.6, we have a distinguished triangle

$$
\tilde{E} \rightarrow \mathbb{D}(E) \rightarrow T[-1] \rightarrow \tilde{E}[1],
$$

where $T$ is a sheaf supported in dimension zero and $\tilde{E}$ is $\nu_{\alpha,-\beta}$-semistable. By our choice of $\alpha$ and the fact that $\operatorname{ch}_{\leqslant 2}(\tilde{E})=(1,0,-d)$, we can use Lemma 2.5 to see that $\tilde{E}$ is a slope semistable sheaf and, thus, must be an ideal sheaf as claimed.

Proof of Theorem 4.2. Lemma 4.5 already shows that Conjecture 4.1 holds for $C$ if $\mathcal{I}_{C}$ is destabilized by a quotient $\mathcal{I}_{C} \rightarrow \mathcal{O}(-f-4)[1]$. Therefore, by Lemmas 4.7 and 4.8, we can assume 


\section{Derived CATEgories AND THE Genus of SPACE CURVES}

that $\mathcal{I}_{C}$ is destabilized by an exact sequence $0 \rightarrow E \rightarrow \mathcal{I}_{C} \rightarrow G(-f-5) \rightarrow 0$, where $\operatorname{ch}_{0}(G)=-1$, $\operatorname{ch}_{1}(G)=0$, and $h^{2}(E(k-1))=0$. We set $y=\operatorname{ch}_{2}(G)$.

Next, we will check that $G(-f-5)$ is stable for $\alpha \gg 0$ by showing that $W\left(\mathcal{I}_{C}, G(-f-5)\right)$ is above or equal to the largest wall for $G(-f-5)$. We have $\operatorname{ch}_{1}^{-f-4}(G(-f-5))=1$. Therefore, there is no wall for $G(-f-5)$ that intersects the vertical line $\beta=-f-4$. However, by assumption, $W\left(\mathcal{I}_{C}, G(-f-5)\right)$ is larger than $W\left(\mathcal{I}_{C}, O(-f-4)\right)$, which contains the point $\alpha=0, \beta=-f-4$.

Note that the conjecture is equivalent to

$$
\chi\left(\mathcal{I}_{C}(k-1)\right) \leqslant\left(\begin{array}{c}
f-k+4 \\
3
\end{array}\right) .
$$

The equality $h^{0}\left(\mathcal{I}_{C}(k-1)\right)=0$ implies $h^{0}(E(k-1))=0$. Thus, we get

$$
\chi(E(k-1)) \leqslant-h^{1}(E(k-1)) \leqslant-h^{0}(G(k-f-6)) .
$$

By Lemma 4.9 , we know that $\mathbb{D}(G)$ fits into a distinguished triangle

$$
\mathcal{I}_{C^{\prime}} \rightarrow \mathbb{D}(G) \rightarrow T[-1] \rightarrow \mathcal{I}_{C^{\prime}}[1]
$$

where $C^{\prime} \subset \mathbb{P}^{3}$ is a one-dimensional subscheme and $T$ is a sheaf supported in dimension zero. Therefore, we get

$$
\begin{aligned}
\chi\left(\mathcal{I}_{C}(k-1)\right) & =\chi(E(k-1))+\chi(G(k-f-6)) \leqslant-h^{0}(G(k-f-6))+\chi(G(k-f-6)) \\
& \leqslant h^{2}(G(k-f-6))=\operatorname{ext}^{2}(\mathbb{D}(G), \mathcal{O}(k-f-6)[1]) \\
& =h^{0}(\mathbb{D}(G)(f-k+2))=h^{0}\left(\mathcal{I}_{C^{\prime}}(f-k+2)\right) .
\end{aligned}
$$

The degree of $C^{\prime}$ is given by

$$
y \geqslant A(k, f+1)-d \geqslant A(k, f+1)-B(k, f)=f-k+3 .
$$

Hence, we can use Corollary 4.4 to obtain

$$
\chi\left(\mathcal{I}_{C}(k-1)\right) \leqslant h^{0}\left(\mathcal{I}_{C^{\prime}}(f-k+2)\right) \leqslant\left(\begin{array}{c}
f-k+4 \\
3
\end{array}\right) .
$$

\subsection{An example}

We finish the article by giving a proof of Conjecture 4.1 for $d=A(k, 2 k-11)$ and $k \geqslant 31$.

Proposition 4.10. Let $C \subset \mathbb{P}^{3}$ be an integral curve of degree $d=A(k, 2 k-11)$ such that $H^{0}\left(\mathcal{I}_{C}(k-1)\right)=0$ for some integer $k \geqslant 31$, and let $\operatorname{ch}\left(\mathcal{I}_{C}\right)=(1,0,-d, e)$. Then

$$
e \leqslant E(d, k) \text {. }
$$

Lemma 4.11. Assume the hypothesis of Proposition 4.10, and assume $e>E(d, k)$.

(i) We have

$$
\begin{aligned}
A(k, 2 k-11) & =k^{2}-7 k+19, \\
E\left(k^{2}-7 k+19, k\right) & =k^{3}-\frac{21}{2} k^{2}+\frac{87}{2} k-65 .
\end{aligned}
$$

(ii) The ideal sheaf $\mathcal{I}_{C}$ is destabilized via a short exact sequence $0 \rightarrow E \rightarrow \mathcal{I}_{C} \rightarrow G(7-2 k) \rightarrow 0$, where $\operatorname{ch}_{1}(G)=0$ and $E$ is a reflexive sheaf of rank two.

(iii) If $\operatorname{ch}(G)=(-1,0, y, z)$, then $0 \leqslant y \leqslant 6$.

(iv) We have $\chi(G(6-k)) \leqslant \frac{1}{6} k^{3}-4 k^{2}-k y+\frac{1}{2} y^{2}+\frac{191}{6} k+\frac{17}{2} y-84$.

(v) We have $h^{2}(E(k-1)) \leqslant\left(\begin{array}{c}y-2 \\ 2\end{array}\right)$. 


\section{E. MACRÌ AND B. SCHMIDT}

Proof. (i) This part is a simple computation.

(ii) Let $\rho_{Q}$ be the radius of the semidisk $Q_{\alpha, \beta}\left(\mathcal{I}_{C}\right) \leqslant 0$. Using $e>k^{3}-\frac{21}{2} k^{2}+\frac{87}{2} k-65$, we get

$$
\rho_{Q}^{2}-\frac{\Delta\left(\mathcal{I}_{C}\right)}{24}>\frac{8 k^{6}-168 k^{5}+903 k^{4}+1402 k^{3}-35817 k^{2}+147360 k-229600}{48\left(k^{2}-7 k+19\right)^{2}}>0 .
$$

By Lemma 2.4, any destabilizing subobject of $\mathcal{I}_{C}$ has rank at most two. Moreover, this shows that the region where $Q_{\alpha, \beta}\left(\mathcal{I}_{C}\right)<0$ is non-empty. Thus, $\mathcal{I}_{C}$ has to be destabilized at some point, and by Lemma 3.12, any destabilizing subobject $E$ has to be a reflexive sheaf.

Assume $\operatorname{ch}_{0}(E)=1$. Then $E$ is a line bundle. Using $e>k^{3}-\frac{21}{2} k^{2}+\frac{87}{2} k-65$ we obtain

$$
Q_{0,-k}\left(\mathcal{I}_{C}\right)<-7 k^{3}+125 k^{2}-674 k+1444<0 \text {. }
$$

Using $H^{0}\left(\mathcal{I}_{C}(k-1)\right)=0$, we see that there are no lines bundles destabilizing $\mathcal{I}_{C}$ as subobjects. We showed that $E$ is a rank two reflexive sheaf. Let $G(-x)$ be the quotient, where $\operatorname{ch}_{1}(G)=0$ for some $x \in \mathbb{Z}$.

Next, we check that $x=2 k-7$. We know that the wall $W\left(E, \mathcal{I}_{C}\right)$ has to be outside the region $Q_{\alpha, \beta}\left(\mathcal{I}_{C}\right)<0$. Thus, for any $\beta \in \mathbb{R}$ with $Q_{0, \beta}\left(\mathcal{I}_{C}\right)<0$, we get

$$
0<\operatorname{ch}_{1}^{\beta}(G)=x+\beta<\operatorname{ch}_{1}^{\beta}\left(\mathcal{I}_{C}\right)=-\beta .
$$

The inequality $e>k^{3}-\frac{21}{2} k^{2}+\frac{87}{2} k-65$ can be used to show that

$$
\begin{gathered}
Q_{0,-2 k+8}\left(\mathcal{I}_{C}\right)<-2 k^{3}+50 k^{2}-308 k+756 \leqslant 0, \\
Q_{0,-k+3}\left(\mathcal{I}_{C}\right)<-k^{3}+38 k^{2}-245 k+616 \leqslant 0 .
\end{gathered}
$$

In particular, $x=2 k-7$ holds. Let $s_{Q}$ be the center of the semidisk $Q_{\alpha, \beta}\left(\mathcal{I}_{C}\right) \leqslant 0$ on the $\beta$-axis. The inequality $s\left(E, \mathcal{I}_{C}\right) \leqslant s_{Q}$ together with $e>k^{3}-\frac{21}{2} k^{2}+\frac{87}{2} k-65$ implies

$$
0 \leqslant y<\frac{9\left(3 k^{2}-23 k+64\right)}{4\left(k^{2}-7 k+19\right)}<7 .
$$

(iii) By Proposition 3.2, we have $z \leqslant \frac{1}{2} y(y+1)$. The statement then follows from the HirzebruchRiemann-Roch theorem. Recall that the Todd class of $\mathbb{P}^{3}$ is given by $\left(1,2, \frac{11}{6}, 1\right)$.

(iv) This is a direct consequence of Theorem 1.10 applied to $E(k-1)$.

Proof of Proposition 4.10. Note that for $d=A(k, 2 k-11)$, Conjecture 4.1 is equivalent to

$$
\chi\left(\mathcal{I}_{C}(k-1)\right) \leqslant\left(\begin{array}{c}
f-k+4 \\
3
\end{array}\right)+h(d)=\left(\begin{array}{c}
k-7 \\
3
\end{array}\right) .
$$

Assume $e>E(d, k)$; then Lemma 4.11 implies that $\mathcal{I}_{C}$ is destabilized by a quotient $\mathcal{I}_{C} \rightarrow$ $G(7-2 k)$, where $\operatorname{ch}_{1}(G)=0$. We have $0 \leqslant y \leqslant 6$ for $y=\operatorname{ch}_{2}(G)$. Then

$$
\chi\left(\mathcal{I}_{C}(k-1)\right) \leqslant \frac{1}{6} k^{3}-4 k^{2}-k y+\frac{1}{2} y^{2}+\frac{191}{6} k+\frac{17}{2} y-84+\left(\begin{array}{c}
y-2 \\
2
\end{array}\right) \leqslant\left(\begin{array}{c}
k-7 \\
3
\end{array}\right) .
$$

\section{ACKNOWLEDGEMENTS}

We would like to thank Roman Bezrukavnikov for originally proposing this question during a talk by the first author, and Arend Bayer, Izzet Coskun, Patricio Gallardo, Martí Lahoz, Luigi Lombardi, César Lozano Huerta, John Ottem, Giuseppe Pareschi, Paolo Stellari, and Xiaolei Zhao for very useful discussions. We also thank the referee for useful suggestions. Parts of the paper were written while the first author was holding a Poincaré Chair from the Institut Henri 


\section{DERIVED CATEGORIES AND THE GENUS OF SPACE CURVES}

Poincaré and the Clay Mathematics Institute. The authors would also like to acknowledge the following institutions: Institut Henri Poincaré, Northeastern University, and University of Texas.

\section{REFERENCES}

AB13 D. Arcara and A. Bertram, Bridgeland-stable moduli spaces for $K$-trivial surfaces (with an appendix by Max Lieblich), J. Eur. Math. Soc. 15 (2013), no. 1, 1-38; doi:10.4171/JEMS/354.

$\mathrm{BBE}^{+} 97$ E. Ballico, G. Bolondi, P. Ellia, and R. M. Mirò-Roig, Curves of maximum genus in range A and stick-figures, Trans. Amer. Math. Soc. 349 (1997), no. 11, 4589-4608; doi:10.1090/ S0002-9947-97-01917-X.

BMS16 A. Bayer, E. Macrì, and P. Stellari, The space of stability conditions on abelian threefolds, and on some Calabi-Yau threefolds, Invent. Math. 206 (2016), no. 3, 869-933; doi:10.1007/ s00222-016-0665-5.

BMT14 A. Bayer, E. Macrì, and Y. Toda, Bridgeland stability conditions on threefolds I: BogomolovGieseker type inequalities, J. Algebraic Geom. 23 (2014), no. 1, 117-163; doi:10.1090/ S1056-3911-2013-00617-7.

Bog79 F. A. Bogomolov, Holomorphic tensors and vector bundles on projective manifolds, Math. USSR Izv. 13 (1979), no. 3, 499-555; doi:10.1070/IM1979v013n03ABEH002076.

Bri08 T. Bridgeland, Stability conditions on K3 surfaces, Duke Math. J. 141 (2008), no. 2, 241-291; doi:10.1215/S0012-7094-08-14122-5.

BvdB03 A. Bondal and M. van den Bergh, Generators and representability of functors in commutative and noncommutative geometry, Mosc. Math. J. 3 (2003), no. 1, 1-36; doi:10.17323/ 1609-4514-2003-3-1-1-36.

CCD93 L. Chiantini, C. Ciliberto, and V. Di Gennaro, The genus of projective curves, Duke Math. J. 70 (1993), no. 2, 229-245; doi:10.1215/S0012-7094-93-07003-2.

CG90 F. Catanese and L. Gœttsche, d-very-ample line bundles and embeddings of Hilbert schemes of 0-cycles, Manuscripta Math. 68 (1990), no. 3, 337-341; doi:10.1007/BF02568768.

CH16 I. Coskun and J. Huizenga, The ample cone of moduli spaces of sheaves on the plane, Algebr. Geom. 3 (2016), no. 1, 106-136; doi:10.14231/AG-2016-005.

Deb94 O. Debarre, Degrees of curves in abelian varieties, Bull. Soc. Math. France 122 (1994), no. 3, 343-361; doi:10.24033/bsmf. 2236.

El191 P. Ellia, Sur le genre maximal des courbes gauches de degré d non sur une surface de degré $s-1$, J. reine angew. Math. 413 (1991), 78-87; doi:10.1515/crll.1991.413.78.

ES92 P. Ellia and R. Strano, Sections planes et majoration du genre des courbes gauches, Complex Projective Geometry (Trieste, 1989/Bergen, 1989), London Math. Soc. Lecture Note Ser., vol. 179 (Cambridge Univ. Press, Cambridge, 1992), 157-174; doi:10.1017/ CB09780511662652.012.

Gie79 D. Gieseker, On a theorem of Bogomolov on Chern classes of stable bundles, Amer. J. Math. 101 (1979), no. 1, 77-85; doi:10.2307/2373939.

GP78 L. Gruson and C. Peskine, Genre des courbes de l'espace projectif, Algebraic Geometry (Proc. Sympos., Univ. Troms $\varnothing$, Troms $\varnothing$, 1977), Lecture Notes in Math., vol. 687 (Springer, Berlin, 1978), 31-59; doi:10.1007/BFb0062927.

GP83 , Postulation des courbes gauches, Algebraic Geometry - Open Problems (Ravello, 1982), Lecture Notes in Math., vol. 997 (Springer, Berlin, 1983), 218-227; doi:10.1007/BFb0061646.

Hal82 G. Halphen, Mémoire sur la classification des courbes gauches algébriques, J. École Polyt. 52 (1882), 1-200; doi:10.2307/2373939.

Har80 J. Harris, The genus of space curves, Math. Ann. 249 (1980), no. 3, 191-204; doi:10.1007/ BF01363895. 


\section{E. MACRì AND B. SchmidT}

Har82a Curves in projective space, Séminaire de Mathématiques Supérieures, vol. 85 (Presses de l'Univ. de Montréal, Montreal, Que.), 1982.

Har77 R. Hartshorne, Algebraic geometry, Grad. Texts in Math., vol. 52 (Springer-Verlag, New YorkHeidelberg, 1977); doi:10.1007/978-1-4757-3849-0.

Har82b R. Hartshorne, Stable reflexive sheaves. II, Invent. Math. 66 (1982), no. 1, 165-190; doi:10. $1007 / \mathrm{BF} 01404762$.

Har87 On the classification of algebraic space curves. II, Algebraic Geometry, Bowdoin, 1985 (Brunswick, Maine, 1985), Proc. Sympos. Pure Math., vol. 46 (Amer. Math. Soc., Providence, RI, 1987), 145-164.

Har88 Stable reflexive sheaves. III, Math. Ann. 279 (1988), no. 3, 517-534; doi:10.1007/ BF01456285.

HH88 R. Hartshorne and A. Hirschowitz, Nouvelles courbes de bon genre dans l'espace projectif, Math. Ann. 280 (1988), no. 3, 353-367; doi:10.1007/BF01456330.

HRS96 D. Happel, I. Reiten, and S. O. Smalø, Tilting in abelian categories and quasitilted algebras, Mem. Amer. Math. Soc. 120 (1996), no. 575; doi:10.1090/memo/0575.

Kos18 N. Koseki, Stability conditions on product threefolds of projective spaces and Abelian varieties, Bull. Lond. Math. Soc. 50 (2018), no. 2, 229-244; doi:10.1112/blms.12132.

Lan04 A. Langer, Semistable sheaves in positive characteristic, Ann. of Math. 159 (2004), no. 1, 251276; doi:10.4007/annals.2004.159.251.

Li19 C. Li, Stability conditions on Fano threefolds of Picard number 1, J. Eur. Math. Soc. 21 (2019), no. 3, 709-726; doi:10.4171/JEMS/848.

LN16 L. Lombardi and W. Niu, Theta-regularity of curves and Brill-Noether loci, Math. Res. Lett. 23 (2016), no. 6, 1761-1787; doi:10.4310/MRL.2016.v23.n6.a9.

Mac14a A. Maciocia, Computing the walls associated to Bridgeland stability conditions on projective surfaces, Asian J. Math. 18 (2014), no. 2, 263-279; doi:10.4310/AJM.2014.v18.n2.a5.

Mac14b E. Macrì, A generalized Bogomolov-Gieseker inequality for the three-dimensional projective space, Algebra Number Theory 8 (2014), no. 1, 173-190; doi:10.2140/ant.2014.8.173.

MP15 A. Maciocia and D. Piyaratne, Fourier-Mukai transforms and Bridgeland stability conditions on abelian threefolds, Algebr. Geom. 2 (2015), no. 3, 270-297; doi:10.14231/AG-2015-012.

MP16_ Fourier-Mukai transforms and Bridgeland stability conditions on abelian threefolds II, Internat. J. Math. 27 (2016), no. 1, 1650007; doi:10.1142/S0129167X16500075.

MR83 V.B. Mehta and A. Ramanathan, Homogeneous bundles in characteristic p, Algebraic Geometry - Open Problems (Ravello, 1982), Lecture Notes in Math., vol. 997 (Springer, Berlin, 1983), 315-320; doi:10.1007/BFb0061650.

MS19 C. Martinez and B. Schmidt, Bridgeland stability on blow ups and counterexamples (with appendix by Omprokash Das), Math. Z. 292 (2019), no. 3-4, 14951510; doi:10.1007/ s00209-018-2149-3.

PP08 G. Pareschi and M. Popa, Castelnuovo theory and the geometric Schottky problem, J. reine angew. Math. 615 (2008), 25-44; doi:10.1515/CRELLE.2008.008.

Rei78 M. Reid, Bogomolov's theorem $c_{1}^{2} \leqslant 4 c_{2}$, Proceedings of the International Symposium on Algebraic Geometry (Kyoto Univ., Kyoto, 1977) (Kinokuniya Book Store, Tokyo, 1978), 623-642.

Sch14 B. Schmidt, A generalized Bogomolov-Gieseker inequality for the smooth quadric threefold, Bull. Lond. Math. Soc. 46 (2014), no. 5, 915-923; doi:10.1112/blms/bdu048.

Sch15_, Bridgeland stability on threefolds - some wall crossings, 2015, arXiv:1509.04608.

Sch17 C Counterexample to the generalized Bogomolov-Gieseker inequality for threefolds, Int. Math. Res. Not. 2017 (2017), no. 8, 2562-2566; doi:10.1093/imrn/rnw122.

Str90 R. Strano, Plane sections of curves of $\mathbb{P}^{3}$ and a conjecture of Hartshorne and Hirschowitz, Rend. Sem. Mat. Univ. Politec. Torino 48 (1990), no. 4, 511-527; available at http://www. seminariomatematico. unito.it/rendiconti/cartaceo/48-4/511.pdf. 


\section{Derived CATEgories AND THE Genus of SPACE CURVES}

Sun18 H. Sun, Arithmetic genus of integral space curves, Czechoslovak Math. J. 68 (2018), no. 4, 1079-1089; doi:10.21136/CMJ.2017.0093-17.

Sun19 , Tilt-stability, vanishing theorems and Bogomolov-Gieseker type inequalities, Adv. Math. 347 (2019), 677-707; doi:10.1016/j.aim.2019.02.026.

Tra14 R. Tramel, The genus of projective curves on complete intersection surfaces, 2014, arXiv: 1408.3543

Xia18 B. Xia, Hilbert scheme of twisted cubics as a simple wall-crossing, Trans. Amer. Math. Soc. 370 (2018), no. 8, 5535-5559; doi:10.1090/tran/7150.

Emanuele Macrì emanuele.macri@universite-paris-saclay.fr

Northeastern University, Department of Mathematics, 360 Huntington Avenue, Boston, MA 02115-5000, USA

Current address: Université Paris-Saclay, CNRS, Laboratoire de mathématiques d'Orsay, 91405, Orsay, France

Benjamin Schmidt bschmidt@math.uni-hannover.de

The University of Texas at Austin, Department of Mathematics, 2515 Speedway, RLM 8.100, Austin, TX 78712, USA

Current address: Gottfried Wilhelm Leibniz Universität Hannover, Institut für Algebraische Geometrie, Welfengarten 1, 30167 Hannover, Germany 